\title{
STABILITY ANALYSIS \\ OF THE NONLINEAR GALERKIN METHOD
}

\author{
R. TEMAM
}

\begin{abstract}
Our object in this article is to describe some numerical schemes for the approximation of nonlinear evolution equations, and to study the stability of the schemes. Spatial discretization can be performed by either spectral or pseudospectral methods, finite elements or finite differences; time discretization is done by two-level schemes, partly or fully explicit.

The algorithms that we present stem from the study of the evolution equations from the dynamical systems point of view. They are based on a differentiated treatment of the small and large wave lengths, and they are particularly adapted to the integration of such equations on large intervals of time.
\end{abstract}

\section{INTRODUCTION}

The long-time integration of dissipative evolution equations has emerged as a new type of problem in numerical analysis, and its appearance is due to the considerable increase in computing power that we have seen during the last years.

Much effort has been devoted in the past to the approximation of evolution equations on finite intervals of time or, in the dynamically simple case where the solution converges to a steady (stationary) state as time goes to infinity. However, in many physically relevant situations the solutions to a dissipative evolution equation do not converge to a stationary solution. They rather remain time-dependent and they converge to a compact attractor that encompasses the nonlinear dynamics. Hence, and whether this is addressed implicitly or explicitly, the numerical integration of the evolution equation is then closely related to the approximation of the attractor that may be a complicated set, even a fractal.

Inertial algorithms are new integration algorithms that are based on the approximation of the attractor by smooth, simple manifolds (namely the approximate inertial manifolds). These algorithms will be recalled below but, at this point, let us point out that one of their main features is a differentiated treatment of small and large wave lengths. An inertial manifold is an exact law of dependence of small wave lengths in terms of the large ones, the small wave

Received April 3, 1990. $35 \mathrm{~A} 40$.

1980 Mathematics Subject Classification (1985 Revision). Primary 65F10, 65F35, 65N20, 
lengths being "slaved" by the large ones. An approximate inertial manifold provides an approximate form of such a law. The decompositions of functions into small and large wave lengths components will be described in detail in $\S 2$. They appear naturally when Fourier series or other spectral expansions are used. With finite elements they appear naturally with the utilization of hierarchical bases; finally, for finite differences they hinge upon the utilization of the incremental unknowns presented in [24] (see §2).

As far as the space discretization is concerned, inertial algorithms, including the nonlinear Galerkin method, have been introduced and studied in $[7,6,18$, $19,24,15,16]$ and other references quoted therein. In the present article we address the question of both time and space discretizations. In particular, we will emphasize the study of the stability analysis of such schemes and, as we will see, a striking fact is that the stability is mostly governed by the large wave lengths. This is an indication, broadly confirmed by the numerical tests, that these schemes are more advantageous than regular schemes for which the time step is commanded by both large and small wave lengths: the time step which is allowed for explicit or semi-implicit schemes is larger.

Strictly speaking, this article does not provide a practical justification of the algorithms presented here, except for an analysis of stability. A full justification of these algorithms relies, on the theoretical side, on results concerning the attractors and their approximation (see, for instance, $[9,8,7,17,22,23]$, and the books $[12,25])$; on the practical side it relies on the results of numerical tests, some of which are reported elsewhere (see [5, 6, 13, 14]), others being in progress and will be reported in the future.

This paper is organized as follows. In $\S 1$ we present the differential equations that we approximate by time discretization. These equations are finitedimensional differential equations corresponding to some (spatial) discretization of partial differential equations; however, since the emphasis here is on stability analysis, we do not describe the continuous problem. In $\S 2$ we introduce the decomposition of the unknown function into small and large wave lengths and describe them explicitly for spectral methods and, in space dimension one, for finite elements and finite differences. For finite differences this involves the utilization of the incremental unknowns. In $\S 3$ we present the various schemes based on the decomposition of the space, and in $\S 4$ we perform the stability analysis of these schemes by energy methods. Although the energy method provides only sufficient stability conditions, it is the only method adapted to a complete mathematical analysis, and it gives some indication on the necessary stability conditions. Finally, in $\S 5$ we present several applications and make explicit the results of the previous sections. In a subsequent work we will study similar schemes based on the decomposition of the unknowns into several (more than two) scales of wave lengths.

\section{THE CONTINUOUS PROBLEM}

We denote by $V_{h}$ a finite-dimensional vector space which is endowed with two scalar products and norms denoted $((\cdot, \cdot))_{h},\|\cdot\|_{h},(\cdot, \cdot)_{h},|\cdot|_{h}$. In the 
applications, $V_{h}$ will be typically the approximation of a Sobolev-type space, $\|\cdot\|_{h}$ will be a discrete Sobolev norm, and $|\cdot|_{h}$ will be a discrete $L^{2}$-norm. This space $V_{h}$ belongs to a family of spaces $V_{h}, h \in \mathscr{H}$, which approximate an infinite-dimensional space $V$ in the limit $h \rightarrow 0$; however, these spaces and the parameter $h$ (the discretization parameter) will not appear explicitly henceforth, except in some specific situations: for example, we will make explicit the dependence of certain constants on $h$. We will denote by $c_{i}$ some (positive) absolute constants independent of $h$, and by $S_{i}=S_{i}(h)$ some constants depending on $h$ and which usually converge to 0 as $h \rightarrow 0$. It is assumed, for instance, that the norms on $V_{h}$ are related in the following manner:

$$
\left|u_{h}\right|_{h} \leq c_{1}\left\|u_{h}\right\|_{h}, \quad S_{1}(h)\left\|u_{h}\right\|_{h} \leq\left|u_{h}\right|_{h} \quad \forall u_{h} \in V_{h} .
$$

We are also given

-a bilinear continuous form $a_{h}(\cdot, \cdot)$ on $V_{h}$ which satisfies

$$
\left|a_{h}\left(u_{h}, v_{h}\right)\right| \leq c_{2}\left\|u_{h}\right\|_{h}\left\|v_{h}\right\|_{h} \quad \forall u_{h}, v_{h} \in V_{h},
$$

-a trilinear continuous form $b_{h}(\cdot, \cdot, \cdot)$ on $V_{h}$, satisfying

$$
\begin{gathered}
b_{h}\left(u_{h}, v_{h}, v_{h}\right)=0 \quad \forall u_{h}, v_{h} \in V_{h}, \\
\left|b_{h}\left(u_{h}, v_{h}, w_{h}\right)\right| \leq c_{3}\left|u_{h}\right|_{h}^{1 / 2}\left\|u_{h}\right\|_{h}^{1 / 2}\left\|v_{h}\right\|_{h}\left|w_{h}\right|_{h}^{1 / 2}\left\|w_{h}\right\|_{h}^{1 / 2} \\
\forall u_{h}, v_{h}, w_{h} \in V_{h},
\end{gathered}
$$

-a bilinear continuous form $d_{h}(\cdot, \cdot)$ on $V_{h}$, such that

$$
\begin{gathered}
\left|d_{h}\left(u_{h}, v_{h}\right)\right| \leq c_{4}\left\|u_{h}\right\|_{h}\left|v_{h}\right|_{h} \quad \forall u_{h}, v_{h} \in V_{h}, \\
a_{h}\left(u_{h}, u_{h}\right)+d_{h}\left(u_{h}, u_{h}\right) \geq c_{5}\left\|u_{h}\right\|^{2} \quad \forall u_{h} \in V_{h} .
\end{gathered}
$$

We are then interested in the initial value problem:

Find a function $u_{h}: \mathbb{R}_{+} \rightarrow V_{h}$ such that

$$
\begin{aligned}
& \frac{d}{d t}\left(u_{h}, v_{h}\right)_{h}+a_{h}\left(u_{h}, v_{h}\right)+b_{h}\left(u_{h}, u_{h}, v_{h}\right)+d_{h}\left(u_{h}, v_{d}\right) \\
& \quad=\left(f_{h}, v_{h}\right) \quad \forall v_{h} \in V_{h},
\end{aligned}
$$

Here, $u_{0 h}$ is given in $V_{h}$ and $f_{h}$ is given in $L^{\infty}\left(\mathbb{R}_{+} ; V_{h}\right)$. Since $V_{h}$ is finitedimensional, it is clear, thanks to (1.3), that the initial value problem (1.7), (1.8) has a unique solution defined for all time,

$$
u_{h} \in L^{\infty}\left(\mathbb{R}_{+} ; V_{h}\right)
$$

more precisely, $u_{h}$ is bounded independently of $h$, from $\mathbb{R}_{+}$into the space $V_{h}$ endowed with the norm $|\cdot|_{h}$.

There are many physically relevant equations which provide, by space discretization, an evolution equation of the form (1.7), the hypotheses (1.2)-(1.6) 
being satisfied. Among the most important equations, one can quote the NavierStokes equations in space dimension two, the Boussinesq equations (dimension two), or the Kuramoto-Sivashinsky equations. The details for these equations can be found in [25] or [19]; some related equations are recalled in $\S 5$.

Our object hereafter is to investigate new forms of time discretizations of (1.7) that take advantage of suitable decompositions of the space $V_{h}$.

\section{INCREMENTAL UNKNOWNS}

We fix a value $h_{1}$ of the parameter $h$ but, for the sake of simplicity in the notations, we will denote the corresponding space $V_{h}$ and its elements $u_{h}, v_{h}, \ldots$. We consider then another space $V_{h}$ corresponding to the value $h=h_{2}$ of the parameter and such that $V_{h_{2}}$ is included in $V_{h}\left(=V_{h_{1}}\right)$. We denote by $W_{h}$ a supplementary of $V_{h_{2}}$ into $V_{h}$ :

$$
V_{h}=V_{h_{2}} \oplus W_{h} .
$$

The elements of $V_{h_{2}}$ will be denoted $y_{h}, \hat{y}_{h}, \ldots$, and those of $W_{h}$ will be denoted $z_{h}, \hat{z}_{h}, \ldots$. Any $u_{h} \in V_{h}$ can be uniquely written as

$$
u_{h}=y_{h}+z_{h}, \quad y_{h} \in V_{h_{2}}, \quad z_{h} \in W_{h} \text {. }
$$

For reasons which will become clear hereafter, $y_{h}$ will be called the large wave lengths or large eddies component of $u_{h}$, and $z_{h}$ will be called the small wave lengths or small eddies component of $u_{h}$. Examples of decompositions (2.1) will be given below; before doing that, we state the main hypotheses related to the decomposition (2.1) of $V_{h}$. We first require an enhanced Cauchy-Schwarz inequality of the form

$$
\left|\left(\left(y_{h}, z_{h}\right)\right)\right| \leq(1-\delta)\left\|y_{h}\right\|_{h}\left\|z_{h}\right\|_{h} \quad \forall y_{h} \in V_{h_{2}}, \forall z_{h} \in W_{h},
$$

where $0<\delta \leq 1$ is independent of $h$. We also assume that

$$
\left|z_{h}\right|_{h} \leq S_{2}(h)\left\|z_{h}\right\|_{h} \quad \forall z_{h} \in W_{h},
$$

where $S_{2}(h) \rightarrow 0$ as $h \rightarrow 0$.

We now give three important decompositions of the form (2.1).

(i) Spectral discretization. This corresponds to the case where the spaces $V_{h}$ are all subspaces of a Hilbert space $V$ (scalar product $((\cdot, \cdot))$, norm $\|\cdot\|$ ), the forms $a_{h}\left(u_{h}, v_{h}\right)$ are just the restrictions to $V_{h}$ of a bilinear continuous symmetric coercive form $a(u, v)$ on $V$. Also, $V$ is continuously imbedded and dense in another Hilbert space $H$ (scalar product $(\cdot, \cdot)$, norm $|\cdot|)$. Then

$$
\left\|u_{h}\right\|_{h}=\left\|u_{h}\right\|, \quad\left|u_{h}\right|_{h}=\left|u_{h}\right| \quad \forall u_{h} \in V_{h} .
$$

Associated with $a, V$, and $H$ is an unbounded self-adjoint operator $A$ in $H$ with domain $D(A) \subset V$, which possesses a basis $w_{j}, j \in \mathbb{N}$, which is orthonormal in $H$ and orthogonal in $V$ :

$$
A w_{j}=\lambda_{j} w_{j} \quad \forall j \in \mathbb{N}, 0<\lambda_{1} \leq \lambda_{2} \leq \cdots, \lambda_{j} \rightarrow \infty \text { as } j \rightarrow \infty .
$$


Here, given $m=m_{1} \in \mathbb{N}$, we set $h=1 / m_{1}$ and

$$
V_{h}=\operatorname{Span}\left\{w_{1}, \ldots, w_{m_{1}}\right\}
$$

and (1.7) results as the Galerkin approximation of some infinite-dimensional problem in the space $V$.

Concerning the decomposition (2.1), we consider another integer $m_{2} \in \mathbb{N}$, $m_{2}<m_{1}$, and we set

$$
V_{h_{2}}=\operatorname{Span}\left\{w_{1}, \ldots, w_{m_{2}}\right\}, \quad W_{h}=\operatorname{Span}\left\{w_{m_{2}+1}, \ldots, w_{m_{1}}\right\}
$$

In this case, $V_{h}$ and $W_{h}$ are orthogonal in $V_{h}$ (scalar product $((\cdot, \cdot))_{h}=$ $((\cdot, \cdot)))$ and $(2.3)$ is satisfied with $\delta=1$. For $(2.4)$ we observe that, for every $z_{h} \in W_{h}, z_{h}=\sum_{j=m_{2}+1}^{m_{1}} \xi_{j} w_{j}$, we have

$$
\begin{aligned}
\left|z_{h}\right|_{h}^{2} & =\left\|\sum_{j=m_{2}+1}^{m_{1}} \xi_{j} w_{j}\right\|^{2}=\sum_{j=m_{2}+1}^{m_{1}}\left|\xi_{j}\right|^{2} \lambda_{j} \\
& \geq \lambda_{m_{2}+1} \sum_{j=m_{2}+1}^{m_{1}}\left|\xi_{j}\right|^{2}=\lambda_{m_{2}+1}\left|z_{h}\right|_{h}^{2} .
\end{aligned}
$$

Hence,

$$
S_{2}=\left(\lambda_{m_{2}+1}\right)^{-1 / 2}
$$

When in the continuous problem, $a$ and $V$ are associated with an elliptic boundary value problem with space periodic boundary conditions, it is well known that the $w_{j}$ are related to Fourier series and, depending on $b_{h},(1.7)$ is a spectral or a pseudospectral approximation [11,2] of the continuous problem. In this case, it is clear that, in (2.2), $y_{h}$ corresponds to the large wave lengths and $z_{h}$ corresponds to the small wave lengths.

(ii) Finite elements. We restrict ourselves to the simplest situation: onedimensional case with piecewise linear elements. More involved situations will be discussed below and elsewhere.

The spaces $V_{h}$ are all subspaces of the Hilbert space $V=H_{0}^{1}(\Omega), \Omega=$ $(0, L), L>0$, and they are all endowed with the same scalar product

$$
\left(\left(u_{h}, v_{h}\right)\right)_{h}=\left(\left(u_{h}, v_{h}\right)\right)=\int_{0}^{L} \frac{d u_{h}}{d x} \frac{d v_{h}}{d x} d x
$$

The scalar products $(\cdot, \cdot)_{h}$ are those of $L^{2}(0, L)$ :

$$
\left(u_{h}, v_{h}\right)_{h}=\left(u_{h}, v_{h}\right)=\int_{0}^{L} u_{h} v_{h} d x
$$

We set $h_{1}=h=1 / 2 N$ and $h_{2}=2 h_{1}=1 / N, N \in \mathbb{N}$. Then $V_{h}$ is the space of real continuous functions on $(0, L)$, which vanish at 0 and $L$ and are linear on the intervals $[j h,(j+1) h], j=0, \ldots, 2 N-1$. The space $V_{h_{2}}=V_{2 h}$ is defined in the same manner, but the functions in $V_{2 h}$ are linear on the intervals 
$[2 j h, 2(j+1) h], j=0, \ldots, N-1$. The choice of the factor 2 for the ratio $h_{2} / h_{1}$ is totally arbitrary; any other integer would be suitable as well.

The nodal basis of $V_{h}$ consists of the functions $w_{j, h}$ of $V_{h}$ which are equal to 1 at $j h$ and to 0 at the points $i h, i, j=1, \ldots, 2 N-1, i \neq j$. Similarly, the nodal basis of $V_{2 h}$ consists of the functions $w_{j, 2 h}$ of $V_{2 h}$ which are equal to 1 at $2 j h$ and to 0 at the points $2 i h, i, j=1, \ldots, N-1, i \neq j$. The hierarchical basis of $V_{h}$ consists of the union of the basis of $V_{2 h}$ and of a basis of $W_{h}$ : namely that consisting of the $w_{j, h}, j=2 i+1, i=0, \ldots, N-1$. If $u_{h}$ belongs to $V_{h}$, its decomposition in the nodal basis reads

$$
u_{h}=\sum_{j=1}^{2 N-1} u_{h}(j h) w_{j, h},
$$

while its decomposition in the hierarchical basis reads

$$
u_{h}=\sum_{j=1}^{N-1} u_{h}(2 j h) w_{j, 2 h}+\sum_{i=0}^{N-1} \bar{u}_{h}((2 i+1) h) w_{2 i+1, h} .
$$

Here, $\bar{u}_{h}((2 i+1) h)$ is the incremental value of $u_{h}$ :

$$
\bar{u}_{h}((2 i+1) h)=u_{h}((2 i+1) h)-\frac{1}{2}\left(u_{h}(2 i h)+u_{h}((2 i+2) h)\right) .
$$

Note also that the first sum in (2.8) corresponds to the component $y_{h} \in V_{h_{2}}$ of $V_{h}$, while the second sum corresponds to the component $z_{h} \in W_{h}$. It is clear that if $h$ is small and $u_{h}$ is the restriction to $V_{h}$ of a smooth function $u$ in $H_{0}^{1}(0, L),{ }^{1}$ then $y_{h}$ will be of the same order as $u_{h}$, while $z_{h}$ will be smaller by a factor $h^{2}$ : this justifies the terminology concerning small and large wave lengths.

It is easy to check that each function $y_{h} \in V_{h_{2}}$ is orthogonal in $V$ to each function $z_{h} \in W_{h}{ }^{2}$ Hence, (2.3) is obviously satisfied with $\delta=1$. The verification of (2.4) has been performed in [19]; we have

$$
S_{2}(h)=h / \sqrt{3} \text {. }
$$

The verification of $(1.1)$ is standard; in particular, for $S_{1}(h)$, setting $\xi_{i}=$ $u_{h}(i h)$, we see that

$$
\int_{0}^{L}\left(\frac{d u_{h}}{d x}\right)^{2} d x=\frac{1}{2} \sum_{i=0}^{2 N-1}\left(\xi_{i+1}-\xi_{i}\right)^{2} .
$$

Similarly,

$$
\int_{0}^{L}\left(u_{h}\right)^{2} d x=\frac{h}{3} \sum_{i=0}^{2 N-1}\left(\xi_{i}^{2}+\xi_{i+1}^{2}+\xi_{i+1} \xi_{i}\right) .
$$

Hence, $S_{1}(h)=h / 2 \sqrt{3}$.

\footnotetext{
${ }^{1}$ i.e., $u_{h}(j h)=u(j h), j=0, \ldots, 2 N$. Then $\bar{u}_{h}((2 i+1) h)=\left(h^{2} / 2\right) u^{\prime \prime}((2 i+1) h)+O\left(h^{3}\right)$.

${ }^{2}$ This property is no longer true in higher dimension.
} 
(iii) Finite differences. We now describe how a decomposition like (2.1) appears in finite differences by using the incremental unknowns introduced in [24].

The form (1.7) of the finite difference discretization of a problem occurs naturally by using the variational formulation of finite differences due to J. Céa [3].

Assume, for instance, that we consider the finite difference approximation of a continuous problem set in $V=H_{0}^{1}(0, L)$. We then set $h=L / 2 N$, $N \in \mathbb{N}$, and $V_{h}$ is the space of step functions that are constant on the intervals $[j h,(j+1) h), j=0, \ldots, 2 N-1$, and that vanish on $[0, h)$ and $[L-h, L)$. The space $V_{h}$ is spanned by the functions $w_{j, h}$ which belong to $V_{h}$, are equal to 1 on $[j h,(j+1) h)$, and to 0 elsewhere, $j=1, \ldots, 2 N-2$ :

$$
u_{h}=\sum_{j=1}^{2 N-2} u_{h}(j h) w_{j, h} \quad \forall u_{h} \in V_{h} .
$$

We call $\left\{w_{j h}\right\}$ the natural basis of $V_{h}$, and we endow this space with the scalar products

$$
\left(\left(u_{h}, v_{h}\right)\right)_{h}=\int_{0}^{L-h} \nabla_{h} u_{h} \nabla_{h} v_{h} d x, \quad\left(u_{h}, v_{h}\right)_{h}=\int_{0}^{L} u_{h} v_{h} d x,
$$

where $\nabla_{h}$ is the forward finite difference operator

$$
\left(\nabla_{h} \varphi\right)(x)=\frac{\varphi(x+h)-\varphi(x)}{h} .
$$

We then set $h_{2}=2 h$ and define in a similar manner the space $V_{h_{2}}=V_{2 h}$, a basis of which consists of the functions $w_{j, 2 h}, j=1, \ldots, N-2$, defined in a similar manner. Here again, the choice of the factor 2 for the ratio $h_{2} / h_{1}$ is totally arbitrary, and we could replace two by any other positive integer. Now we observe that $V_{2 h} \subset V_{h}$ and we complete the decomposition (2.1) by defining $W_{h}$ : this is the space spanned by the functions $w_{2 i+1, h}, i=0, \ldots, N-2$, and $w_{2 N-2, h}$. Any function $u_{h} \in V_{h}$ can be written as

$$
\begin{gathered}
u_{h}=y_{h}+z_{h}, \quad y_{h} \in V_{2 h}, \\
y_{h}=\sum_{j=1}^{N-2} u_{h}(2 j h) w_{j, 2 h}, \\
z_{h}=\sum_{i=0}^{N-2} z_{h}((2 i+1) h) w_{2 i+1, h}+z_{h}((2 N-2) h) w_{2 N-2, h} .
\end{gathered}
$$

Here, for $i=0, \ldots, N-2$,

$$
z_{h}((2 i+1) h)=\bar{u}_{h}((2 i+1) h)=u_{h}((2 i+1) h)-u_{h}(2 i h)
$$

and

$$
z_{h}((2 N-2) h)=u_{h}((2 N-2) h) .
$$


It is clear that if $u_{h}$ is the restriction to $V_{h}$ of a smooth function $u$ in $H_{0}^{1}(0, L),{ }^{3}$ then $y_{h}$ is of the same order as $u_{h}$, while $z_{h}$ will be smaller by a factor $h$ : this justifies the terminology about large and small wave lengths.

The purpose of the following lemmas is to check (2.3) and (2.4).

Lemma 2.1. We have the enhanced Cauchy-Schwarz inequality

$$
\left|\left(\left(y_{h}, z_{h}\right)\right)_{h}\right| \leq \sqrt{\frac{2}{3}}\left\|y_{h}\right\|_{h}\left\|z_{h}\right\|_{h}, \quad \forall y \in V_{2 h}, \forall z_{h} \in W_{h} .
$$

Proof. We must show that

$$
\int_{0}^{L-h} \nabla_{h} y_{h} \nabla_{h} z_{h} d x \leq \sqrt{\frac{2}{3}}\left(\int_{0}^{L-h}\left|\nabla_{h} y_{h}\right|^{2} d x\right)^{1 / 2}\left(\int_{0}^{L-h}\left|\nabla_{h} z_{h}\right|^{2} d x\right)^{1 / 2}
$$

It suffices to show $(2.15)$ with the interval $(0, L)$ replaced by each of the coarsegrid intervals $(2 j h, 2(j+1) h)$.

We first start with a typical interval, i.e., $j=1, \ldots, N-3$. Then $y_{h}=m_{1}$ on $[2 j h, 2(j+1) h)$ and $y_{h}=m_{2}$ on $[2(j+1) h, 2(j+2) h) ; z_{h}=0$ on $[2 j h,(2 j+1) h),=p_{1}$ on $[(2 j+1) h, 2(j+1) h),=0$ on $[2(j+1) h,(2 j+3) h)$, $=p_{2}$ on $[(2 j+3) h, 2(j+2) h)$ (see Figure 2.1$)$.

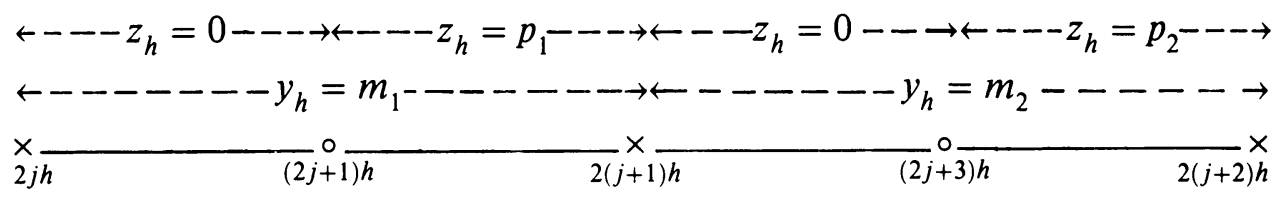

FIGURE 2.1

Values of $y_{h}$ and $z_{h}$ on typical intervals

On $[2 j h,(2 j+1) h)$,

$$
\nabla_{h} y_{h}=0, \quad \nabla_{h} z_{h}=\frac{p_{1}}{h},
$$

and on $[(2 j+1) h, 2(j+1) h)$,

$$
\nabla_{h} y_{h}=\frac{m_{2}-m_{1}}{h}, \quad \nabla_{h} z_{h}=-\frac{p_{1}}{h} .
$$

Thus,

$$
\begin{aligned}
\int_{2 j h}^{2(j+1) h} & \nabla_{h} y_{h} \nabla_{h} z_{h} d x=-\frac{1}{h}\left(m_{2}-m_{1}\right) p_{1} \\
\leq & \frac{1}{\sqrt{2}}\left(\int_{2 j h}^{2(j+1) h}\left|\nabla_{h} y_{h}\right|^{2} d x\right)^{1 / 2}\left(\int_{2 j h}^{2(j+1) h}\left|\nabla_{h} z_{h}\right|^{2} d x\right)^{1 / 2} \\
\quad & \frac{1}{h}\left|m_{2}-m_{1}\right|\left|p_{1}\right| .
\end{aligned}
$$

${ }^{3}$ i.e., $u_{h}(j h)=u(j h), j=0, \ldots, 2 N-2$. 
We consider then the end intervals, $j=0$ and $j=N-2, N-1$. The only difference, for $j=0$, is that $m_{1}=0$; hence $(2.16)$ is still valid. Next we consider the intervals $[L-4 h, L-2 h)$ and $[L-2 h, L-h)$ together; we take into account the fact that $z_{h}$ does not vanish $\left(\right.$ say $\left.=p_{2}\right)$ on $[L-2 h, L-h)$, but $z_{h}=0$ on $[L-h, L)$. We find

$$
\begin{aligned}
\int_{L-4 h}^{L-h} \nabla_{h} y_{h} \nabla_{h} z_{h} d x & =-\frac{1}{h} m_{1}\left(p_{2}-p_{1}\right) \\
& \leq \frac{1}{h} \sqrt{\frac{2}{3}}\left|m_{1}\right|\left(\left(p_{2}-p_{1}\right)^{2}+p_{2}^{2}+p_{1}^{2}\right)^{1 / 2} \\
& =\sqrt{\frac{2}{3}}\left(\int_{L-4 h}^{L-h}\left|\nabla_{h} y_{h}\right|^{2} d x\right)^{1 / 2}\left(\int_{L-4 h}^{L-h}\left|\nabla_{h} z_{h}\right|^{2} d x\right)^{1 / 2} .
\end{aligned}
$$

Since $1 / \sqrt{2} \leq \sqrt{\frac{2}{3}}$, we obtain $(2.14)$.

Lemma 2.2. We have the following strong discrete Poincaré inequality for functions in $W_{h}$ :

$$
\left|z_{h}\right|_{h} \leq S_{2}(h)\left\|z_{h}\right\|_{h} \quad \forall z_{h} \in W_{h}, S_{2}(h)=h .
$$

Proof. As in Lemma 2.1, it suffices to prove the similar inequality on the intervals $[2 j h, 2(j+1) h), j=0, \ldots, N-1$, i.e.,

$$
\int_{2 j h}^{2(j+1) h} z_{h}^{2} d x \leq S_{2}(h)^{2} \int_{2 j h}^{2(j+1) h}\left|\nabla_{h} z_{h}\right|^{2} d x .
$$

Using the same notation as in Lemma 2.1, we see that the integral in the righthand side of (2.18) is equal, for $j=0, \ldots, N-3$, to $2 p_{1}^{2} / h$, while the integral in the left-hand side is equal to $p_{1}^{2} h$; hence $(2.18)$ with $S_{2}(h)=h / \sqrt{2}$. On the interval $(L-4 h, L)$, the integral of $z_{h}^{2}$ is equal to $h\left(p_{1}^{2}+p_{2}^{2}\right)$, and the integral of $\left|\nabla_{h} z_{h}\right|^{2}$ is equal to $(1 / h)\left(\left(p_{1}-p_{2}\right)^{2}+p_{1}^{2}+p_{2}^{2}\right)$. We obtain an inequality similar to (2.18) with $S_{2}(h)=h$, and finally (2.17) is proved.

The proof of $(1.1)$ is standard. Let us recall how the second inequality (1.1) is obtained, and let us derive $S_{1}(h)$. Setting $\xi_{j}=u_{h}(j h)$, we have $u_{h}=$ $\sum_{j=1}^{2 N-2} \xi_{j} w_{j, h}$, and

$$
\begin{gathered}
\left|u_{h}\right|_{h}^{2}=h \sum_{j=1}^{2 N-2} \xi_{j}^{2} \\
\left\|u_{h}\right\|_{h}^{2}=\frac{1}{h} \sum_{j=1}^{2 N-2}\left(\xi_{j+1}-\xi_{j}\right)^{2} \leq \frac{2}{h} \sum_{j=1}^{2 N-2}\left(\xi_{j+1}^{2}+\xi_{j}^{2}\right) \\
=\frac{4}{h} \sum_{j=1}^{2 N-2} \xi_{j}^{2} \quad\left(\text { since } \xi_{0}=\xi_{2 N-1}=0\right) \\
=\frac{4}{h^{2}}\left|u_{h}\right|_{h}^{2} .
\end{gathered}
$$


Thus,

$$
S_{1}(h)=h / 2 \text {. }
$$

Finally, we note the following result, which will be useful.

Lemma 2.3. There holds

$$
\left|y_{h}\right|_{h}=\left|y_{h}\right|_{2 h}, \quad\left\|y_{h}\right\|_{h}^{2}=2\left\|y_{h}\right\|_{2 h}^{2} \quad \forall y_{h} \in V_{2 h}=V_{h_{2}} .
$$

Proof. The first equality (2.20) is obvious, since both norms $|\cdot|_{h}$ and $|\cdot|_{h_{2}}$ are merely the $L^{2}$-norms. For proving the second equality $(2.20)$ we use the same notation as in Lemma 2.1 and observe that $\nabla_{2 h} y_{h}$ is constant on [2jh, $2(j+1) h)$ and equal to $\left(m_{2}-m_{1}\right) / 2 h$, while $\nabla_{h} y_{h}$ is equal to 0 on [ $2 j h$, $(2 j+1) h)$ and to $\left(m_{2}-m_{1}\right) / h$ on $[(2 j+1) h, 2(j+1) h)$. Hence,

$$
\int_{2 j h}^{2(j+1) h}\left|\nabla_{2 h} y_{h}\right|^{2} d x=\frac{\left(m_{2}-m_{1}\right)^{2}}{2 h}=\frac{1}{2} \int_{2 j h}^{2(j+1) h}\left|\nabla_{h} y_{h}\right|^{2} d x,
$$

and (2.20) follows by summation for $j=0, \ldots, N-2$.

Remark 2.1. The space $V_{h_{2}}$ plays exactly the same role as the space $V_{h}\left(=V_{h_{1}}\right)$. Therefore, the analog of hypotheses (1.1)-(1.6) are valid; in particular, the second hypothesis (1.1) reads

$$
S_{1}(2 h)\left\|y_{h}\right\|_{2 h} \leq\left|y_{h}\right|_{2 h} \quad \forall y_{h} \in V_{h_{2}}=V_{2 h} .
$$

In conjunction with (2.19) and (2.20), the inequality (2.21) becomes

$$
\begin{gathered}
\bar{S}_{1}(h)\left\|y_{h}\right\|_{h} \leq\left|y_{h}\right|_{h} \quad \forall y_{h} \in V_{h_{2}}=V_{2 h}, \\
\bar{S}_{1}(h)=S_{1}(2 h) / \sqrt{2} .
\end{gathered}
$$

For the other discretizations (spectral and finite elements), $\left\|y_{h}\right\|_{h_{2}}=\left\|y_{h}\right\|_{h}$ and $\left|y_{h}\right|_{h_{2}}=\left|y_{h}\right|_{h}, \forall y_{h} \in V_{h}$. Thus, (2.22) is still valid, but $\bar{S}_{1}(h)=S_{1}\left(h_{2}\right)$.

\section{THE TIME-DISCRETIZED PROBLEMS}

We now describe the time discretization of equation (1.7); the schemes rely on the decomposition (2.1) of $V_{h}$ and on theoretical work concerning the approximation of attractors.

Four schemes will be proposed. The first two are fully implicit in the linear terms and explicit or partly explicit in the nonlinear terms. The third is also explicit for the nonlinear terms, and for the linear terms it is explicit for the large wave lengths and implicit for the small wave lengths. The fourth differs from the previous ones in that the time evolution of $z$ disappears, as is usual in the nonlinear Galerkin methods [18, 19]. The significance and advantages of the schemes will be discussed below.

Scheme I. The initial data $u_{0 h}$ in (1.8) is decomposed in the form

$$
u_{0 h}=y_{h}^{0}+z_{h}^{0}, \quad y_{h}^{0} \in V_{h_{2}}, \quad z_{h}^{0} \in W_{h} \text {, }
$$


according to (2.2). We then define recursively the sequences of elements $y_{h}^{n} \in$ $V_{h_{2}}, z_{h}^{n} \in W_{h}$, as follows.

When $y_{h}^{n}, z_{h}^{n}$ are known, we define $y_{h}^{n+1} \in V_{h_{2}}$ and $z_{h}^{n+1} \in W_{h}$ by the equations

$$
\begin{aligned}
& \frac{1}{k}\left(y_{h}^{n+1}-y_{h}^{n}, \hat{y}_{h}\right)_{h}+a_{h}\left(y_{h}^{n+1}+z_{h}^{n+1}, \hat{y}_{h}\right)+d_{h}\left(y_{h}^{n+1}+z_{h}^{n+1}, \hat{y}_{h}\right) \\
& \quad+b_{h}\left(y_{h}^{n}, y_{h}^{n}, \hat{y}_{h}\right)+b_{h}\left(y_{h}^{n}, z_{h}^{n}, \hat{y}_{h}\right)+b_{h}\left(z_{h}^{n}, y_{h}^{n}, \hat{y}_{h}\right) \\
& =\left(f_{h}^{n}, \hat{y}_{h}\right)_{h} \quad \forall \hat{y}_{h} \in V_{h_{2}}, \\
& \frac{1}{k}\left(z_{h}^{n+1}-z_{h}^{n}, \hat{z}_{h}\right)_{h}+a_{h}\left(y_{h}^{n+1}+z_{h}^{n+1}, \hat{z}_{h}\right)+d_{h}\left(y_{h}^{n+1}+z_{h}^{n+1}, \hat{z}_{h}\right) \\
& \quad+b_{h}\left(y_{h}^{n}, y_{h}^{n}, \hat{z}_{h}\right)=\left(f_{h}^{n}, \hat{z}_{h}\right)_{h} \quad \forall \hat{z}_{h} \in W_{h} .
\end{aligned}
$$

Here, $k=\Delta t$ is the time step and $f_{h}^{n}$ is a time average of $f_{h}:^{4}$

$$
f_{h}^{n}=\frac{1}{k} \int_{n k}^{(n+1) k} f(t) d t .
$$

Equations (3.2)-(3.3) amount to a linear system for $y_{h}^{n+1}, z_{h}^{n+1}$. Thanks to (1.6), the existence and uniqueness of $y_{h}^{n+1}, z_{h}^{n+1}$ follow readily from LaxMilgram's theorem.

Scheme $\mathbf{I}^{\prime}$. This is a slightly modified version of Scheme I, where the $b$-terms in (3.2) are treated implicitly in $z$. Hence, we replace (3.2) by

$$
\begin{aligned}
& \frac{1}{k}\left(y_{h}^{n+1}-y_{h}^{n}, \hat{y}_{h}\right)_{h}+a_{h}\left(y_{h}^{n+1}+z_{h}^{n+1}, \hat{y}_{h}\right)+d_{h}\left(y_{h}^{n+1}+z_{h}^{n+1}, \hat{y}_{h}\right) \\
& \quad+b_{h}\left(y_{h}^{n}, y_{h}^{n}, \hat{y}_{h}\right)+b_{h}\left(y_{h}^{n}, z_{h}^{n+1}, \hat{y}_{h}\right)+b_{h}\left(z_{h}^{n+1}, y_{h}^{n}, \hat{y}_{h}\right) \\
& =\left(f_{h}^{n}, \hat{y}_{h}\right)_{h} \quad \forall \hat{y}_{h} \in V_{h_{2}},
\end{aligned}
$$

while (3.3) remains unchanged.

Here again, the existence for each $n$ of the pair $y_{h}^{n+1}, z_{h}^{n+1}$, solution of (3.5) and (3.3), follows from Lax-Milgram's theorem, but the proof of this point depends on the a priori estimates which will be proved in $\S 4$; therefore, we postpone the proof of the solvability of (3.5), (3.3) to $\S 4$.

Remark 3.1. Since (3.5), (3.3) is (linearly) implicit in $y_{h}^{n+1}, z_{h}^{n+1}$, with a dependence on $z_{h}^{n+1}$ in the $b$-terms, the solution of (3.5), (3.3) is more difficult than that of (3.2), (3.3). However, we shall see that Scheme $I^{\prime}$ allows a better (larger) time step $k$. Also, the $z_{h}^{n+1}$ component of $u_{h}^{n+1}$ is small; therefore, the dependence of $b$ on $z_{h}^{n+1}$ is rather mild.

\footnotetext{
${ }^{4}$ If $f_{h}$ is smooth, we can take more simply $f_{h}^{n}=f_{h}(n k)$.
} 
Scheme II. We start with the same $y_{h}^{0}, z_{h}^{0}$ as in (3.1) and define recursively the sequences of elements $y_{h}^{n}, z_{h}^{n}$.

When $y_{h}^{n}$ and $z_{h}^{n}$ are known, we define $y_{h}^{n+1} \in V_{h_{2}}$ and $z_{h}^{n+1} \in W_{h}$ by the equations

$$
\begin{aligned}
& \frac{1}{k}\left(y_{h}^{n+1}-y_{h}^{n}, \hat{y}_{h}\right)_{h}+a_{h}\left(y_{h}^{n}+z_{h}^{n+1}, \hat{y}_{h}\right)+d_{h}\left(y_{h}^{n}+z_{h}^{n+1}, \hat{y}_{h}\right) \\
& +b_{h}\left(y_{h}^{n}, y_{h}^{n}, \hat{y}_{h}\right)+b_{h}\left(y_{h}^{n}, z_{h}^{n+1}, \hat{y}_{h}\right)+b_{h}\left(z_{h}^{n+1}, y_{h}^{n}, \hat{y}_{h}\right) \\
& =\left(f_{h}^{n}, \hat{y}_{h}\right)_{h} \quad \forall \hat{y}_{h} \in V_{h_{2}} \text {, } \\
& \frac{1}{k}\left(z_{h}^{n+1}-z_{h}^{n}, \hat{z}_{h}\right)_{h}+a_{h}\left(y_{h}^{n}+z_{h}^{n+1}, \hat{y}_{h}\right)+d_{h}\left(y_{h}^{n}+z_{h}^{n+1}, \hat{y}_{h}\right) \\
& +b_{h}\left(y_{h}^{n}, y_{h}^{n}, \hat{z}_{h}\right)=\left(f_{h}^{n}, \hat{z}_{h}\right)_{h} \quad \forall \hat{z}_{h} \in W_{h} .
\end{aligned}
$$

In fact, we first determine $z_{h}^{n+1}$ by solving (3.7), using (1.6) and Lax-Milgram's theorem. We then determine $y_{h}^{n+1}$ by solving (3.6).

Scheme III. This scheme is a slightly modified form of Scheme II, where the term $z_{h}^{n+1}-z_{h}^{n}$ in (3.7) is removed. The reason for removing this term is that the evolution of the $z$-term (the small structures) is slow and can be neglected (see [7]). ${ }^{5}$ A variation of Scheme II taking this into account consists in computing $z_{h}^{n+1}$ by solving

$$
\begin{aligned}
& a_{h}\left(y_{h}^{n}+z_{h}^{n+1}, \hat{z}_{h}\right)+d_{h}\left(y_{h}^{n}+z_{h}^{n+1}, \hat{z}_{h}\right)+b_{h}\left(y_{h}^{n}, y_{h}^{n}, \hat{z}_{h}\right) \\
& =\left(f_{h}^{n}, \hat{z}_{h}\right) \quad \forall \hat{z}_{h} \in W_{h} .
\end{aligned}
$$

Then $y_{h}^{n+1}$ is determined by (3.6). The existence of a solution $z_{h}^{n+1}$ to (3.8) follows readily from (1.6) and Lax-Milgram's theorem.

Of course, an analog of Schemes I, I' where the $z$-terms are dropped can be presented; we will not do this here.

For all schemes we set

$$
u_{h}^{n}=y_{h}^{n}+z_{h}^{n} \in V_{h} \quad \forall n .
$$

Of course, $u_{h}^{n}$ is expected to be an approximation of $u_{h}(n k)$ in the limit $k \rightarrow 0$.

Remark 3.2. The effective resolution of the linear systems appearing in (3.2), (3.3), (3.5)-(3.7), and (3.8) will be discussed elsewhere and we will, as well, present and discuss the results of numerical experiments.

At this point, one can speculate that the solution of problems (3.3), (3.7), or (3.8) involves almost as many unknowns or is almost as complicated as an implicit or semi-implicit scheme in $V_{h}$, for $u_{h}^{n+1}$. However, besides the theoretical advantages related to the closeness to the attractor that we will not discuss

\footnotetext{
${ }^{5}$ We do not discuss here the validity of this hypothesis, which assumes that the high-frequency modes of $f$ are not too large (see [5]).
} 
here, the two advantages of the schemes of this type are the following:

-Schemes I, I', II, and III yield a better stability condition than a similar scheme in $V_{h}$ (see $\left.\S 5\right)$.

-It will appear from the a priori estimates below that the $z_{h}^{n}$ are small quantities and therefore, the linear systems corresponding to (3.3) or to (3.7) need not be solved with the highest accuracy.

\section{Stability analysis}

Our aim is now to perform a stability analysis for Schemes I-III by using energy methods. Namely, we want to obtain a priori estimates for the $y_{h}^{n}$ and $z_{h}^{n}$ that are independent of $k$ (and $h$ ); we will be able to do so at the price of some conditions on $k$ and $h$, the stability conditions.

Scheme I. For the sake of simplicity, all the indices $h$ will be dropped during the following computations.

We replace $\hat{y}_{h}$ by $2 k y_{h}^{n+1}$ in (3.2) and $\hat{z}_{h}$ by $2 k z_{h}^{n+1}$ in (3.3). We recall that

$$
\begin{aligned}
& 2(a-b, a)=|a|^{2}-|b|^{2}+|a-b|^{2}, \\
& 2(a-b, b)=|a|^{2}-|b|^{2}-|a-b|^{2} .
\end{aligned}
$$

We then obtain (dropping the indices $h$ ):

$$
\begin{aligned}
\left|y^{n+1}\right|^{2} & -\left|y^{n}\right|^{2}+\left|y^{n+1}-y^{n}\right|^{2}+2 k a\left(u^{n+1}, y^{n+1}\right)+2 k d\left(u^{n+1}, y^{n+1}\right) \\
& +2 k b\left(y^{n}, y^{n}, y^{n+1}\right)+2 k b\left(y^{n}, z^{n}, y^{n+1}\right)+2 k b\left(z^{n}, y^{n}, y^{n+1}\right) \\
& =2 k\left(f^{n}, y^{n+1}\right)
\end{aligned}
$$

$$
\begin{aligned}
& \left|z^{n+1}\right|^{2}-\left|z^{n}\right|^{2}+\left|z^{n+1}-z^{n}\right|^{2}+2 k a\left(u^{n+1}, z^{n+1}\right)+2 k d\left(u^{n+1}, z^{n+1}\right) \\
& \quad+2 k b\left(y^{n}, y^{n}, z^{n+1}\right)=2 k\left(f^{n}, z^{n+1}\right) .
\end{aligned}
$$

We add these relations and take into account (1.3) and (1.6); we find

$$
\begin{aligned}
\left(\left|y^{n+1}\right|^{2}\right. & \left.+\left|z^{n+1}\right|^{2}\right)-\left(\left|y^{n}\right|^{2}+\left|z^{n}\right|^{2}\right)+\left|y^{n+1}-y^{n}\right|^{2} \\
& +\left|z^{n+1}-z^{n}\right|^{2}+2 k a\left(u^{n+1}, u^{n+1}\right)+2 k d\left(u^{n+1}, u^{n+1}\right) \\
= & 2 k\left(f^{n}, u^{n+1}\right)-2 k b\left(u^{n}, y^{n}, y^{n+1}-y^{n}\right) \\
& -2 k b\left(y^{n}, z^{n}, y^{n-1}-y^{n}\right)-2 k b\left(y^{n}, y^{n}, z^{n+1}-z^{n}\right) .
\end{aligned}
$$

We write, thanks to (1.1) and (1.6),

$$
\begin{aligned}
2 k a\left(u^{n+1}, u^{n+1}\right) & +2 k d\left(u^{n+1}, u^{n+1}\right) \geq 2 k c_{5}\left\|u^{n+1}\right\|^{2} \\
2 k\left(f^{n}, u^{n+1}\right) & \leq 2 k\left|f^{n}\right|\left|u^{n+1}\right| \leq 2 k c_{1}\left|f^{n}\right|\left\|u^{n+1}\right\| \\
& \leq c_{5} k\left\|u^{n+1}\right\|^{2}+\frac{k c_{1}^{2}}{c_{5}}\left|f^{n}\right|^{2} .
\end{aligned}
$$


Then we majorize the $b$-terms using (1.4), the second inequality (1.1), (2.4), and (2.22):

$$
\begin{aligned}
2 k \mid b & \left(u^{n}, y^{n}, y^{n+1}-y^{n}\right) \mid \\
& \leq 2 k c_{3}\left|u^{n}\right|^{1 / 2}\left\|u^{n}\right\|^{1 / 2}\left\|y^{n}\right\|\left|y^{n+1}-y^{n}\right|^{1 / 2}\left\|y^{n+1}-y^{n}\right\|^{1 / 2} \\
& \leq 2 k c_{3}\left(S_{1} \bar{S}_{1}\right)^{-1 / 2}\left|u^{n}\right|\left\|y^{n}\right\|\left|y^{n+1}-y^{n}\right| \quad(\text { with (1.1) and (2.22)) } \\
& \leq \frac{1}{4}\left|y^{n+1}-y^{n}\right|^{2}+4 k^{2} c_{3}^{2}\left(S_{1} \bar{S}_{1}\right)^{-1}\left|u^{n}\right|^{2}\left\|y^{n}\right\|^{2},
\end{aligned}
$$

where $S_{1}=S_{1}(h)$ and $\bar{S}_{1}=\bar{S}_{1}(h)(\text { see }(2.22))^{6}$. Similarly,

$$
\begin{aligned}
2 k\left|b\left(y^{n}, z^{n}, y^{n+1}-y^{n}\right)\right| & \\
\leq & 2 k c_{3}\left|y^{n}\right|^{1 / 2}\left\|y^{n}\right\|^{1 / 2}\left\|z^{n}\right\|\left|y^{n+1}-y^{n}\right|^{1 / 2}\left\|y^{n+1}-y^{n}\right\|^{1 / 2} \\
\leq & 2 k c_{3}\left(\bar{S}_{1}\right)^{-1}\left|y^{n}\right|\left\|z^{n}\right\|\left|y^{n+1}-y^{n}\right| \\
\leq & \frac{1}{4}\left|y^{n+1}-y^{n}\right|^{2}+4 k^{2} c_{3}^{2}\left(\bar{S}_{1}\right)^{-2}\left|y^{n}\right|^{2}\left\|z^{n}\right\|^{2} \\
2 k \mid & b\left(y^{n}, y^{n}, z^{n+1}-z^{n}\right) \mid \\
& \leq 2 k c_{3}\left|y^{n}\right|^{1 / 2}\left\|y^{n}\right\|^{3 / 2}\left|z^{n+1}-z^{n}\right|^{1 / 2}\left\|z^{n+1}-z^{n}\right\|^{1 / 2} \\
& \leq 2 k c_{3}\left(S_{1} \bar{S}_{1}\right)^{-1 / 2}\left|y^{n}\right|\left\|y^{n}\right\|\left|z^{n+1}-z^{n}\right| \\
& \leq \frac{1}{2}\left|z^{n+1}-z^{n}\right|^{2}+2 k^{2} c_{3}^{2}\left(S_{1} \bar{S}_{1}\right)^{-1}\left|y^{n}\right|^{2}\left\|y^{n}\right\|^{2} .
\end{aligned}
$$

Collecting these inequalities, we obtain

$$
\begin{aligned}
\left(\left|y^{n+1}\right|^{2}\right. & \left.+\left|z^{n+1}\right|^{2}\right)-\left(\left|y^{n}\right|^{2}+\left|z^{n}\right|^{2}\right) \\
& +\frac{1}{2}\left|y^{n+1}-y^{n}\right|^{2}+\frac{1}{2}\left|z^{n+1}-z^{n}\right|^{2}+k c_{5}\left\|u^{n+1}\right\|^{2} \\
\leq & \frac{k}{c_{1}^{2} c_{5}}\left|f^{n}\right|^{2}+4 k^{2} c_{3}^{2}\left(S_{1} \bar{S}_{1}\right)^{-1}\left|u^{n}\right|^{2}\left\|y^{n}\right\|^{2} \\
& +4 k^{2} c_{3}^{2}\left(\bar{S}_{1}\right)^{-2}\left|y^{n}\right|^{2}\left\|z^{n}\right\|^{2}+2 k^{2} c_{3}^{2}\left(S_{1} \bar{S}_{1}\right)^{-1}\left|y^{n}\right|^{2}\left\|y^{n}\right\|^{2} .
\end{aligned}
$$

Because of (2.3),

$$
\begin{aligned}
\left\|y_{h}+z_{h}\right\|_{h}^{2} & =\left\|y_{h}\right\|_{h}^{2}+\left\|z_{h}\right\|_{h}^{2}+2\left(\left(y_{h}, z_{h}\right)\right)_{h} \\
& \geq\left\|y_{h}\right\|_{h}^{2}+\left\|z_{h}\right\|_{h}^{2}-2(1-\delta)\left\|y_{h}\right\|_{h}\left\|z_{h}\right\|_{h}, \\
& \geq \delta\left(\left\|y_{h}\right\|_{h}^{2}+\left\|z_{h}\right\|_{h}^{2}\right) .
\end{aligned}
$$

Also,

$$
\left|u_{h}\right|_{h}^{2}=\left|y_{h}+z_{h}\right|_{h}^{2} \leq 2\left(\left|y_{h}\right|_{h}^{2}+\left|z_{h}\right|_{h}^{2}\right),
$$

\footnotetext{
${ }^{6}$ Although we dropped the indices $h$, we recall that $\left|y^{n+1}-y^{n}\right|=\left|y_{h}^{n+1}-y_{h}^{n}\right|_{h}$ and $\left\|y^{n+1}-y^{n}\right\|$ $=\left\|y_{h}^{n+1}-y_{h}^{n}\right\|_{h}$.
} 
and (4.4) yields

$$
\begin{aligned}
\left(\left|y^{n+1}\right|^{2}\right. & \left.+\left|z^{n+1}\right|^{2}\right)-\left(\left|y^{n}\right|^{2}+\left|z^{n}\right|^{2}\right)+\frac{1}{2}\left|y^{n+1}-y^{n}\right|^{2} \\
& +\frac{1}{2}\left|z^{n+1}-z^{n}\right|^{2}+k c_{5} \delta\left(\left\|y^{n+1}\right\|^{2}+\left\|z^{n+1}\right\|^{2}\right) \\
\leq & \frac{k c_{1}^{2}}{c_{5}}\left|f^{n}\right|^{2}+k \Lambda\left(\left|y^{n}\right|^{2}+\left|z^{n}\right|^{2}\right)\left(\left\|y^{n}\right\|^{2}+\left\|z^{n}\right\|^{2}\right)
\end{aligned}
$$

with

$$
\Lambda=2 k c_{3}^{2}\left(9\left(S_{1} \bar{S}_{1}\right)^{-1}+2\left(\bar{S}_{1}\right)^{-2}\right)
$$

We now prove

Lemma 4.1. We assume that

$$
k \leq 2 c_{1}^{2} / c_{5} \delta
$$

and

$$
\Lambda=2 k c_{3}^{2}\left(9\left(S_{1}(h) \bar{S}_{1}(h)\right)^{-1}+2\left(\bar{S}_{1}(h)\right)^{-2}\right) \leq c_{5} \delta / 2 M,
$$

with

$$
\begin{aligned}
& \bar{M}=\left(1+\frac{1}{2} k c_{5} S_{1}(h)^{-2}\right)\left(\left|y_{h}^{0}\right|_{h}^{2}+\left|z_{h}^{0}\right|_{h}^{2}\right), \\
& M=\bar{M}+\frac{4 c_{1}^{4}}{c_{5}^{2} \delta}|f|_{L^{\infty}\left(\mathbb{R}_{+} ; V_{h},|\cdot|_{h}\right)}^{2} .
\end{aligned}
$$

Then, for every $n \geq 0$,

$$
\mu_{n}=\left|y_{h}^{n}\right|_{h}^{2}+\left|z_{h}^{n}\right|_{h}^{2} \leq M .
$$

Proof. We proceed by induction; (4.10) is obviously true for $n=0$. Assuming it has been proved up to order $n$, we want to establish it at order $n+1$.

We observe that the induction hypothesis and (4.9)-(4.11) imply that $\Lambda \mu_{n} \leq$ $\frac{1}{2} c_{5} \delta$. We then set

$$
\xi^{n}=\left|y^{n}\right|^{2}+\left|z^{n}\right|^{2}+\frac{1}{2} k c_{5} \delta\left(\left\|y^{n}\right\|^{2}+\left\|z^{n}\right\|^{2}\right)
$$

and we infer from (4.6) that

$$
\xi^{n+1}-\xi^{n}+\frac{1}{2} k c_{5} \delta\left(\left\|y^{n+1}\right\|^{2}+\left\|z^{n+1}\right\|^{2}\right) \leq \frac{k c_{1}^{2}}{c_{5}}|f|_{L^{\infty}}^{2}
$$

where

$$
|f|_{L^{\infty}}^{2}=\left|f_{h}\right|_{L^{\infty}\left(\mathbb{R}_{+} ; V_{h},|\cdot|_{h}\right)}^{2} .
$$


But, by (1.1),

$$
\begin{aligned}
\| y^{n+1} & \left\|^{2}+\right\| z^{n+1} \|^{2} \\
& \geq \frac{1}{2 c_{1}^{2}}\left(\left|y^{n+1}\right|^{2}+\left|z^{n+1}\right|^{2}\right)+\frac{1}{2}\left(\left\|y^{n+1}\right\|^{2}+\left\|z^{n+1}\right\|^{2}\right) \\
& \geq \frac{1}{2 c_{1}^{2}}\left(\left(\left|y^{n+1}\right|^{2}+\left|z^{n+1}\right|^{2}\right)+c_{1}^{2}\left(\left\|y^{n+1}\right\|^{2}+\left\|z^{n+1}\right\|^{2}\right)\right) \\
& \geq \frac{1}{2 c_{1}^{2}} \xi^{n+1} \quad(\text { thanks to }(4.8))
\end{aligned}
$$

and (4.13) yields

$$
\begin{gathered}
\left(1+\frac{k c_{5} \delta}{4 c_{1}^{2}}\right) \xi^{n+1} \leq \xi^{n}+\frac{k c_{1}^{2}}{c_{5}}|f|_{L^{\infty}}^{2} \\
\xi^{n+1} \leq\left(1+\frac{k c_{5} \delta}{4 c_{1}^{2}}\right)^{-1} \xi^{n}+\frac{k c_{1}^{2}}{c_{5}}\left(1+\frac{k c_{5} \delta}{4 c_{1}^{2}}\right)^{-1}|f|_{L^{\infty}}^{2} .
\end{gathered}
$$

The analogous relations are also valid for the previous values of $n$, and thus

$$
\begin{aligned}
\left(1+\frac{k c_{5} \delta}{4 c_{1}^{2}}\right)^{-1} \xi^{n} & \leq\left(1+\frac{k c_{5} \delta}{4 c_{1}^{2}}\right)^{-2} \xi^{n-1}+\frac{k c_{1}^{2}}{c_{5}}\left(1+\frac{k c_{5} \delta}{4 c_{1}^{2}}\right)^{-2}|f|_{L^{\infty}}^{2}, \\
& \vdots \\
\left(1+\frac{k c_{5} \delta}{4 c_{1}^{2}}\right)^{-n} \xi^{1} & \leq\left(1+\frac{k c_{5} \delta}{4 c_{1}^{2}}\right)^{-n-1} \xi^{0}+\frac{k c_{1}^{2}}{c_{5}}\left(1+\frac{k c_{5} \delta}{4 c_{1}^{2}}\right)^{-n-1}|f|_{L^{\infty}}^{2}
\end{aligned}
$$

By adding all these relations, we obtain

$$
\begin{gathered}
\xi^{n+1} \leq\left(1+\frac{k c_{5} \delta}{4 c_{1}^{2}}\right)^{-n-1} \xi^{0}+\frac{4 c_{1}^{4}}{c_{5}^{2} \delta}|f|_{L^{\infty}}^{2} \\
\xi^{n+1} \leq \xi^{0}+\frac{4 c_{1}^{4}}{c_{5}^{2} \delta}|f|_{L^{\infty}}^{2}
\end{gathered}
$$

In particular,

$$
\begin{aligned}
\left|y^{n+1}\right|^{2} & +\left|z^{n+1}\right|^{2} \\
& \leq\left|y^{0}\right|^{2}+\left|z^{0}\right|^{2}+\frac{1}{2} k c_{5} \delta\left(\left\|y^{0}\right\|^{2}+\left\|z^{0}\right\|^{2}\right)+\frac{4 c_{1}^{4}}{c_{5}^{2} \delta}|f|_{L^{\infty}}^{2} \\
& \leq\left(1+\frac{1}{2} k c_{5} \delta S_{1}^{-2}\right)\left(\left|y^{0}\right|^{2}+\left|z^{0}\right|^{2}\right)+\frac{4 c_{1}^{4}}{c_{5}^{2} \delta}|f|_{L^{\infty}}^{2}=M
\end{aligned}
$$

The induction is complete and Lemma 4.1 is proved. 
Remark 4.1. For the study of convergence, in passing to the limit $k \rightarrow 0, h \rightarrow$ 0 , the bound (4.10), (4.11) is effective provided

$$
k S_{1}(h)^{-2} \text { remains bounded }
$$

(by any finite number). Condition (4.9) requires $\Lambda=\Lambda(k, h)$ to be smaller than a specific constant.

We associate with the elements $y_{h}^{n}, z_{h}^{n}$, and $u_{h}^{n}$ some approximating step functions defined as follows:

$$
\begin{array}{ll}
y_{h}: \mathbb{R}_{+} \rightarrow V_{h_{1}}, & y_{h}(t)=y_{h}^{n}, \quad t \in[n k,(n+1) k), \\
z_{h}: \mathbb{R}_{+} \rightarrow W_{h}, & z_{h}(t)=z_{h}^{n}, \quad t \in[n k,(n+1) k), \\
u_{h}: \mathbb{R}_{+} \rightarrow V_{h_{2}}, & u_{h}(t)=y_{h}(t)+z_{h}(t) .
\end{array}
$$

Lemma 4.1 can be interpreted as follows:

Under the hypotheses (4.8)-(4.10),

$$
\left|y_{h}\right|_{L^{\infty}\left(\mathbb{R}_{+} ; V_{h},|\cdot|_{h}\right)}^{2}+\left|z_{h}\right|_{L^{\infty}\left(\mathbb{R}_{+} ; V_{h},|\cdot|_{h}\right)}^{2} \leq M,
$$

and with (4.18),

$$
\begin{aligned}
& u_{h}, y_{h} \text {, and } z_{h} \text { remain bounded in } L^{\infty}\left(\mathbb{R}_{+} ; V_{h},|\cdot|_{h}\right), \\
& \text { as } k \text { and } h \rightarrow 0 \text {. }
\end{aligned}
$$

With the same hypotheses we can derive further estimates on $y_{h}, z_{h}$, and $u_{h}$.

Let $T>0$ be fixed, let $N k \leq T<(N+1) k$, and add the relations (4.13) for $n=0, \ldots, N$. We find

$$
\begin{aligned}
\xi^{N+1} & +\frac{1}{2} k c_{5} \delta \sum_{n=0}^{N}\left(\left\|y^{n+1}\right\|^{2}+\left\|z^{n+1}\right\|^{2}\right) \leq \xi^{0}+\frac{k N c_{1}^{2}}{c_{5}}|f|_{L^{\infty}}^{2} \\
& \leq\left(1+\frac{1}{2} k c_{5} \delta S_{1}^{-2}\right)\left(\left|y_{h}^{0}\right|^{2}+\left|z_{h}^{0}\right|^{2}\right)+\frac{T c_{1}^{2}}{c_{5}}|f|_{L^{\infty}}^{2} \\
& \leq \bar{M}+\frac{T c_{1}^{2}}{c_{5}}|f|_{L^{\infty}\left(\mathbb{R}_{+} ; V_{h},|\cdot|_{h}\right)}^{2} .
\end{aligned}
$$

Hence,

$$
\begin{aligned}
& \text { under hypotheses }(4.8)-(4.10), \\
& \left|y_{h}\right|_{L^{2}\left(0, T ; V_{h},\|\cdot\|_{h}\right)}^{2}+\left|z_{h}\right|_{L^{2}\left(0, T ; V_{h},\|\cdot\|_{h}\right)}^{2} \leq M^{\prime}, \\
& M^{\prime}=\frac{2}{c_{5} \delta}\left(\bar{M}+\frac{T c_{1}^{2}}{c_{5}}|f|_{L^{\infty}\left(\mathbb{R}_{+} ; V_{h},|\cdot|_{h}\right)}^{2}\right)
\end{aligned}
$$

or, with (4.18),

$$
\begin{aligned}
& u_{h}, y_{h}, z_{h} \text { remain bounded in } L^{2}\left(0, T ; V_{h},\|\cdot\|_{h}\right) \\
& \forall T>0, \text { as } k \text { and } h \rightarrow 0 .
\end{aligned}
$$


Scheme $I^{\prime}$. The analysis is very similar to that of Scheme I, but some simplifications and improvements occur.

We replace $\hat{y}_{h}$ by $2 k y_{h}^{n+1}$ in (3.5) and $\hat{z}_{h}$ by $2 k z_{h}^{n+1}$ in (3.6); after adding the corresponding relations, we obtain the analog of (4.3):

$$
\begin{aligned}
& \left(\left|y^{n+1}\right|^{2}+\left|z^{n+1}\right|^{2}\right)-\left(\left|y^{n}\right|^{2}+\left|z^{n}\right|^{2}\right)+\left|y^{n+1}-y^{n}\right|^{2} \\
& \quad+\left|z^{n+1}-z^{n}\right|^{2}+2 k a\left(u^{n+1}, u^{n+1}\right)+2 k d\left(u^{n+1}, u^{n+1}\right) \\
& =2 k\left(f^{n}, u^{n+1}\right)-2 k b\left(y^{n}, y^{n}, y^{n+1}-y^{n}\right) \\
& \quad-2 k b\left(z^{n+1}, y^{n}, y^{n+1}-y^{n}\right)-2 k b\left(y^{n}, z^{n+1}, y^{n+1}-y^{n}\right) .
\end{aligned}
$$

We reproduce the computations following (4.3):

$$
\begin{aligned}
& 2 k a\left(u^{n+1}, u^{n+1}\right)+2 k d\left(u^{n+1}, u^{n+1}\right) \geq 2 k c_{5}\left\|u^{n+1}\right\|^{2}, \\
& 2 k\left(f^{n}, u^{n+1}\right) \leq k c_{5}\left\|u^{n+1}\right\|^{2}+\frac{k c_{1}^{2}}{c_{5}}\left|f^{n}\right|^{2}, \\
& 2 k\left|b\left(y^{n}, y^{n}, y^{n+1}-y^{n}\right)\right| \\
& \leq 2 k c_{3}\left|y^{n}\right|^{1 / 2}\left\|y^{n}\right\|^{3 / 2}\left|y^{n+1}-y^{n}\right|^{1 / 2}\left\|y^{n+1}-y^{n}\right\|^{1 / 2} \\
& \leq 2 k c_{3}\left(\bar{S}_{1}\right)^{-1}\left|y^{n}\right|\left\|y^{n}\right\|\left|y^{n+1}-y^{n}\right| \\
& \leq \frac{1}{4}\left|y^{n+1}-y^{n}\right|^{2}+4 k^{2} c_{3}^{2}\left(\bar{S}_{1}\right)^{-2}\left|y^{n}\right|^{2}\left\|y^{n}\right\|^{2},
\end{aligned}
$$

$$
\begin{aligned}
& 2 k\left|b\left(z^{n+1}, y^{n}, y^{n+1}-y^{n}\right)\right| \\
& \leq 2 k c_{3}\left|z^{n+1}\right|^{1 / 2}\left\|z^{n+1}\right\|^{1 / 2}\left\|y^{n}\right\|\left|y^{n+1}-y^{n}\right|^{1 / 2}\left\|y^{n+1}-y^{n}\right\|^{1 / 2} \\
& \leq 2 k c_{3}\left(S_{2} / \bar{S}_{1}\right)^{1 / 2}\left(\bar{S}_{1}\right)^{-1}\left|y^{n}\right|\left\|z^{n+1}\right\|\left|y^{n+1}-y^{n}\right| \quad(\text { with }(2.4) \text { and }(2.22)) \\
& \leq \frac{1}{4}\left|y^{n+1}-y^{n}\right|^{2}+4 k^{2} c_{3}^{2}\left(S_{2} / \bar{S}_{1}\right)\left(\bar{S}_{1}\right)^{-2}\left|y^{n}\right|^{2}\left\|z^{n+1}\right\|^{2} \\
& 2 k\left|b\left(y^{n}, z^{n+1}, y^{n+1}-y^{n}\right)\right| \\
& \leq 2 k c_{3}\left|y^{n}\right|^{1 / 2}\left\|y^{n}\right\|^{1 / 2}\left\|z^{n+1}\right\|\left|y^{n+1}-y^{n}\right|^{1 / 2}\left\|y^{n+1}-y^{n}\right\|^{1 / 2} \\
& \leq 2 k c_{3}\left(\bar{S}_{1}\right)^{-2}\left|y^{n}\right|\left\|z^{n+1}\right\|\left|y^{n+1}-y^{n}\right| \\
& \leq \frac{1}{4}\left|y^{n+1}-y^{n}\right|^{2}+4 k^{2} c_{3}^{2}\left(\bar{S}_{1}\right)^{-2}\left|y^{n}\right|^{2}\left\|z^{n+1}\right\|^{2}
\end{aligned}
$$

With these inequalities, (4.24) yields

$$
\begin{aligned}
\left(\left|y^{n+1}\right|^{2}\right. & \left.+\left|z^{n+1}\right|^{2}\right)-\left(\left|y^{n}\right|^{2}+\left|z^{n}\right|^{2}\right) \\
& +\frac{1}{4}\left|y^{n+1}-y^{n}\right|^{2}+\left|z^{n+1}-z^{n}\right|^{2}+k c_{5}\left\|u^{n+1}\right\|^{2} \\
\leq & \frac{k c_{1}^{2}}{c_{5}}\left|f^{n}\right|^{2}+4 k^{2} c_{3}^{2}\left(\bar{S}_{1}\right)^{-2}\left(1+\frac{S_{2}}{\bar{S}_{1}}\right)\left|y^{n}\right|^{2}\left\|z^{n+1}\right\|^{2} \\
& +4 k^{2} c_{3}^{2}\left(\bar{S}_{1}\right)^{-2}\left|y^{n}\right|^{2}\left\|y^{n}\right\|^{2} .
\end{aligned}
$$


Lemma 4.2. We assume (4.8) and

$$
\begin{gathered}
4 k c_{3}^{2}\left(\bar{S}_{1}(h)\right)^{-2}\left(1+S_{2}(h) / \bar{S}_{1}(h)\right) M_{1} \leq \frac{1}{4} c_{5} \delta, \\
\left.M_{1}=\bar{M}+\frac{8 c_{1}^{4}}{c_{5}^{2} \delta}|f|_{L^{\infty}}^{2} \mathbb{R}_{+} ; V_{h},|\cdot|_{h}\right)
\end{gathered}
$$

with $\bar{M}$ as in (4.10). Then for every $n \geq 0$,

$$
\mu_{n}=\left|y_{h}^{n}\right|_{h}^{2}+\left|z_{h}^{n}\right|_{h}^{2} \leq M_{1}
$$

Proof. The proof is similar to that of Lemma 4.1, and we consider the same expression $\xi^{n}$ as in (4.12).

We proceed by induction; (4.27) is true for $n=0$, and, assuming that it has been proved up to order $n$, we want to prove it at order $n+1$. From (4.5), (4.25),

$$
\begin{gathered}
\xi^{n+1}-\xi^{n}+\frac{1}{4} k c_{5} \delta\left(\left\|y^{n+1}\right\|^{2}+\left\|z^{n+1}\right\|^{2}\right) \\
\leq \frac{k c_{1}^{2}}{c_{5}}\left|f^{n+1}\right|^{2} \leq \frac{k c_{1}^{2}}{c_{5}}|f|_{L^{\infty}}^{2} .
\end{gathered}
$$

Using (4.14), we find, in place of (4.15),

$$
\xi^{n+1} \leq\left(1+\frac{k c_{5} \delta}{8 c_{1}^{2}}\right)^{-1} \xi^{n}+\frac{k c_{1}^{2}}{c_{5}}\left(1+\frac{k c_{5} \delta}{8 c_{1}^{2}}\right)^{-1}|f|_{L^{\infty}}^{2}
$$

With the analogous relations valid for the previous values of $n$, we obtain

$$
\xi^{n+1} \leq\left(1+\frac{k c_{5} \delta}{8 c_{1}^{2}}\right)^{n-1} \xi^{0}+\frac{8 c_{1}^{4}}{c_{5}^{2} \delta}|f|_{L^{\infty}}^{2}
$$

and we conclude as in Lemma 4.1.

Remark 4.2. Under the hypotheses of Lemma 4.2, (4.28) implies the coercivity of the linear system (3.5), (3.3) defining $y_{h}^{n+1}, z_{h}^{n+1}$ for Scheme I'; hence, the solvability (yet unproved) of this scheme by the Lax-Milgram theorem.

Remark 4.3. The stability conditions (4.9) and (4.26) will be discussed and compared in $\S 5$.

We define the functions $y_{h}, z_{h}$, and $u_{h}$ as in (4.19), and from Lemma 4.2 we infer that

under the hypotheses (4.8) and (4.26),

$$
\begin{aligned}
& \left|y_{h}\right|_{L^{\infty}\left(\mathbb{R}_{+} ; V_{h},|\cdot|_{h}\right)}^{2}+\left|z_{h}\right|_{L^{\infty}\left(\mathbb{R}_{+} ; V_{h},|\cdot|_{h}\right)}^{2} \leq M_{1}, \\
& \left|y_{h}\right|_{L^{2}\left(0, T ; V_{h},\|\cdot\|_{h}\right)}^{2}+\left|z_{h}\right|_{L^{2}\left(0, T ; V_{h},\|\cdot\|_{h}\right)}^{2} \leq 2 M^{\prime} \quad \forall T>0
\end{aligned}
$$

$\left(M_{1}, M^{\prime}\right.$ as in (4.26) and (4.22)), and with (4.18),

$u_{h}, y_{h}$, and $z_{h}$ remain bounded in $L^{\infty}\left(\mathbb{R}_{+} ; V_{h},|\cdot|_{h}\right)$, and in $L^{2}\left(0, T ; V_{h},\|\cdot\|_{h}\right) \forall T>0$, as $k$ and $h \rightarrow 0$. 
Scheme II. We now proceed with the stability analysis for Scheme II; some steps will be the same as for Scheme I, and some will be different.

We replace $\hat{y}_{h}$ by $2 k y_{h}^{n}$ in (3.6) and $\hat{z}_{h}$ by $2 k z_{h}^{n+1}$ in (3.7). Dropping the indices $h$, we find thanks to (1.3):

$$
\begin{aligned}
& \left|y^{n+1}\right|^{2}-\left|y^{n}\right|^{2}-\left|y^{n+1}-y^{n}\right|^{2}+2 k a\left(y^{n}+z^{n+1}, y^{n}\right) \\
& \quad+2 k d\left(y^{n}+z^{n+1}, y^{n}\right)+2 k b\left(y^{n}, z^{n+1}, y^{n}\right)=2 k\left(f^{n}, y^{n}\right), \\
& \left|z^{n+1}\right|^{2}-\left|z^{n}\right|^{2}+\left|z^{n+1}-z^{n}\right|^{2}+2 k a\left(y^{n}+z^{n+1}, z^{n+1}\right) \\
& +2 k d\left(y^{n}+z^{n+1}, z^{n+1}\right)+2 k b\left(y^{n}, y^{n}, z^{n+1}\right)=2 k\left(f^{n}, z^{n+1}\right) .
\end{aligned}
$$

We add these relations, using again (1.3),

$$
\begin{aligned}
& \left(\left|y^{n+1}\right|^{2}+\left|z^{n+1}\right|^{2}\right)-\left(\left|y^{n}\right|^{2}+\left|z^{n}\right|^{2}\right)+\left|z^{n+1}-z^{n}\right|^{2} \\
& \quad+2 k a\left(y^{n}+z^{n+1}, y^{n}+z^{n+1}\right)+2 k d\left(y^{n}+z^{n+1}, y^{n}+z^{n+1}\right) \\
& =2 k\left(f^{n}, y^{n}+z^{n+1}\right)+\left|y^{n+1}-y^{n}\right|^{2} .
\end{aligned}
$$

Thanks to (1.1) and (1.6),

$$
\begin{aligned}
2 k a\left(y^{n}+z^{n+1}, y^{n}+z^{n+1}\right) & +2 k d\left(y^{n}+z^{n+1}, y^{n}+z^{n+1}\right) \geq 2 k c_{5}\left\|y^{n}+z^{n+1}\right\|^{2}, \\
2 k\left(f^{n}, y^{n}+z^{n+1}\right) & \leq 2 k\left|f^{n}\right|\left|y^{n}+z^{n+1}\right| \leq 2 k c_{1}\left|f^{n}\right|\left\|y^{n}+z^{n+1}\right\| \\
& \leq k c_{5}\left\|y^{n}+z^{n+1}\right\|^{2}+\frac{k c_{1}^{2}}{c_{5}}\left|f^{n}\right|^{2} .
\end{aligned}
$$

In order to majorize the term $\left|y^{n+1}-y^{n}\right|^{2}$ in the right-hand side of (4.33), we return to (3.6) and replace $\hat{y}_{h}$ by $k\left(y_{h}^{n+1}-y_{h}^{n}\right)$; thus,

$$
\begin{aligned}
\left|y^{n+1}-y^{n}\right|^{2}= & -k a\left(y^{n}+z^{n+1}, y^{n+1}-y^{n}\right)-k d\left(y^{n}+z^{n+1}, y^{n+1}-y^{n}\right) \\
& -k b\left(y^{n}, y^{n}+z^{n+1}, y^{n+1}-y^{n}\right) \\
& -k b\left(z^{n+1}, y^{n}, y^{n+1}-y^{n}\right)+k\left(f^{n}, y^{n+1}-y^{n}\right) .
\end{aligned}
$$

Because of (1.2), (1.4), and (1.5) we can majorize the right-hand side of this relation by

$$
\begin{aligned}
& k c_{2}\left\|y^{n}+z^{n+1}\right\|\left\|y^{n+1}-y^{n}\right\|+k c_{5}\left\|y^{n}+z^{n+1}\right\|\left|y^{n+1}-y^{n}\right| \\
& \quad+k c_{3}\left|y^{n}\right|^{1 / 2}\left\|y^{n}\right\|^{1 / 2}\left\|y^{n}+z^{n+1}\right\|\left|y^{n+1}-y^{n}\right|^{1 / 2}\left\|y^{n+1}-y^{n}\right\|^{1 / 2} \\
& \quad+k c_{3}\left|z^{n+1}\right|^{1 / 2}\left\|z^{n+1}\right\|^{1 / 2}\left\|y^{n}\right\|\left|y^{n+1}-y^{n}\right|^{1 / 2}\left\|y^{n+1}-y^{n}\right\|^{1 / 2} \\
& \quad+k\left|f^{n}\right|\left|y^{n+1}-y^{n}\right| .
\end{aligned}
$$

In view of (2.4) and (2.22) this expression is bounded by

$$
\begin{aligned}
k\left\{\left(c_{2}\left(\bar{S}_{1}\right)^{-1}+c_{5}\right)\left\|y^{n}+z^{n+1}\right\|\right. & +c_{3}\left(\bar{S}_{1}\right)^{-1}\left|y^{n}\right|\left\|y^{n}+z^{n+1}\right\| \\
& \left.+c_{3}\left(S_{2} / \bar{S}_{1}\right)^{1 / 2}\left(\bar{S}_{1}\right)^{-1}\left|y^{n}\right|\left\|z^{n+1}\right\|+\left|f^{n}\right|\right\}\left|y^{n+1}-y^{n}\right| .
\end{aligned}
$$


Hence, with (4.34),

$$
\begin{aligned}
\left|y^{n+1}-y^{n}\right|^{2} \leq 5 k^{2}\left\{\left(c_{2}^{2}\left(\bar{S}_{1}\right)^{-2}\right.\right. & \left.+c_{5}^{2}+c_{3}^{2}\left(\bar{S}_{1}\right)^{-2}\left|y^{n}\right|^{2}\right)\left\|y^{n}+z^{n+1}\right\|^{2} \\
& \left.+c_{3}^{2}\left(S_{2} / \bar{S}_{1}\right)\left(\bar{S}_{1}\right)^{-2}\left|y^{n}\right|^{2}\left\|z^{n+1}\right\|^{2}+\left|f^{n}\right|^{2}\right\} .
\end{aligned}
$$

With these relations we infer from (4.33):

$$
\begin{aligned}
\left(\left|y^{n+1}\right|^{2}\right. & \left.+\left|z^{n+1}\right|^{2}\right)-\left(\left|y^{n}\right|^{2}+\left|z^{n}\right|^{2}\right)+\left|z^{n+1}-z^{n}\right|^{2}+k c_{5}\left\|y^{n}+z^{n+1}\right\|^{2} \\
\leq & k\left(\frac{c_{1}^{2}}{c_{5}}+5 k\right)|f|_{L^{\infty}}^{2}+5 k^{2}\left(\left(c_{2}^{2}+c_{3}^{2}\left|y^{n}\right|^{2}\right)\left(\bar{S}_{1}\right)^{-2}+c_{5}^{2}\right)\left\|y^{n}+z^{n+1}\right\|^{2} \\
& +5 k^{2} c_{3}^{2}\left(\frac{S_{2}}{\bar{S}_{1}}\right)\left(\bar{S}_{1}\right)^{-2}\left|y^{n}\right|^{2}\left\|z^{n+1}\right\|^{2} .
\end{aligned}
$$

Lemma 4.3. We assume that (4.18) holds and

$$
\begin{gathered}
5 k\left(\left(c_{2}^{2}+c_{3}^{2} M_{2}\right)\left(\bar{S}_{1}(h)\right)^{-2}+c_{5}^{2}\right) \leq \frac{c_{5}}{4}, \\
5 k c_{3}^{2}\left(\frac{S_{2}(h)}{\bar{S}_{1}(h)}\right)\left(\bar{S}_{1}(h)\right)^{-2} M_{2} \leq \frac{c_{5} \delta}{4}, \\
M_{2}=\bar{M}+\frac{8 c_{1}^{4}}{c_{5}^{2} \delta}\left(1+\frac{10 c_{1}^{4}}{\delta}\right)|f|_{L^{\infty}}^{2}\left(\mathbb{R}_{+} ; V_{h},|\cdot|_{h}\right)
\end{gathered}
$$

$\bar{M}$ as in (4.10). Then, for every $n \geq 0$,

$$
\mu_{n}=\left|y_{h}^{n}\right|_{h}^{2}+\left|z_{h}^{n}\right|_{h}^{2} \leq M_{2} .
$$

Proof. Relation (4.38) is obvious for $n=0$. We assume that it has been proved up to order $n$ and we want to prove it at order $n+1$. Because of (4.5) and (4.36), we infer from (4.35),

$$
\begin{aligned}
& \left(\left|y^{n+1}\right|^{2}+\left|z^{n+1}\right|^{2}\right)=\left(\left|y^{n}\right|^{2}+\left|z^{n}\right|^{2}\right)+\left|z^{n+1}-z^{n}\right|^{2}+\frac{3}{4} k c_{5} \delta\left(\left\|y^{n}\right\|^{2}+\left\|z^{n+1}\right\|^{2}\right) \\
& \leq k\left(\frac{c_{1}^{2}}{c_{5}}+5 k\right)|f|_{L^{\infty}}^{2}+5 k^{2} c_{3}^{2}\left(\frac{S_{2}}{\bar{S}_{1}}\right)\left(\bar{S}_{1}\right)^{-2} M_{2}\left\|z^{n+1}\right\|^{2} \\
& \leq k\left(\frac{c_{1}^{2}}{c_{5}}+\frac{10 c_{1}^{2}}{c_{5} \delta}\right)|f|_{L^{\infty}}^{2}+\frac{1}{4} k c_{5} \delta\left\|z^{n+1}\right\|^{2} \quad(\text { by (4.8) and (4.37)) }
\end{aligned}
$$

Hence,

$$
\begin{aligned}
& \left(\left|y^{n+1}\right|^{2}+\left|z^{n+1}\right|^{2}\right)-\left(\left|y^{n}\right|^{2}+\left|z^{n}\right|^{2}\right)+\left|z^{n+1}-z^{n}\right|^{2} \\
& \quad+\frac{1}{2} k c_{5} \delta\left(\left\|y^{n}\right\|^{2}+\left\|z^{n+1}\right\|^{2}\right) \leq k\left(\frac{c_{1}^{2}}{c_{5}}+\frac{10 c_{1}^{2}}{c_{5} \delta}\right)|f|_{L^{\infty}}^{2} .
\end{aligned}
$$

Setting

$$
\xi^{n}=\left|y^{n}\right|^{2}+\left|z^{n}\right|^{2}+\frac{1}{4} k c_{5} \delta\left(\left\|y^{n}\right\|^{2}+\left\|z^{n+1}\right\|^{2}\right),
$$


we rewrite (4.39) in the form

$$
\xi^{n+1}-\xi^{n}+\frac{1}{4} k c_{5} \delta\left(\left\|y^{n}\right\|^{2}+\left\|z^{n+1}\right\|^{2}\right) \leq k\left(\frac{c_{1}^{2}}{c_{5}}+\frac{10 c_{1}^{2}}{c_{5} \delta}\right)|f|_{L^{\infty}}^{2}
$$

As in (4.14), using (1.1) and (4.8),

$$
\begin{aligned}
\left\|y^{n}\right\|^{2}+\left\|z^{n+1}\right\|^{2} & \geq \frac{1}{2 c_{1}^{2}}\left(\left|y^{n}\right|^{2}+\left|z^{n+1}\right|^{2}\right)+\frac{1}{2}\left(\left\|y^{n}\right\|^{2}+\left\|z^{n+1}\right\|^{2}\right) \\
& \geq \frac{1}{2 c_{1}^{2}}\left(\left|y^{n}\right|^{2}+\left|z^{n+1}\right|^{2}\right)+c_{1}^{2}\left(\left\|y^{n}\right\|^{2}+\left\|z^{n+1}\right\|^{2}\right) \geq \frac{1}{2 c_{1}^{2}} \xi^{n+1} .
\end{aligned}
$$

Thus,

$$
\left(1+\frac{k c_{5} \delta}{8 c_{1}^{2}}\right) \xi^{n+1}-\xi^{n} \leq k\left(\frac{c_{1}^{2}}{c_{5}}+\frac{10 c_{1}^{2}}{c_{5} \delta}\right)|f|_{L^{\infty}}^{2}
$$

and also, for $j=0, \ldots, n$,

$$
\left(1+\frac{k c_{5} \delta}{8 c_{1}^{2}}\right)^{-(n-j)} \xi^{j+1} \leq\left(1+\frac{k c_{5} \delta}{8 c_{1}^{2}}\right)^{-(n+1-j)}\left(\xi^{j}+k\left(\frac{c_{1}^{2}}{c_{5}}+\frac{10 c_{1}^{2}}{c_{5} \delta}\right)|f|_{L^{\infty}}^{2}\right) \text {. }
$$

By adding these relations for $j=0, \ldots, n$, we obtain

$$
\begin{gathered}
\xi^{n+1} \leq\left(1+\frac{k c_{5} \delta}{8 c_{1}^{2}}\right)^{-(n+1)} \xi^{0}+\frac{8 c_{1}^{4}}{c_{5}^{2} \delta}\left(1+\frac{10}{\delta}\right)|f|_{L^{\infty}}^{2} \\
\xi^{n+1} \leq\left(\left|y^{0}\right|^{2}+\left|z^{0}\right|^{2}\right)+\frac{1}{4} k c_{5} \delta\left(\left\|y^{0}\right\|^{2}+\left\|z^{0}\right\|^{2}\right) \\
+\frac{8 c_{1}^{4}}{c_{5}^{2} \delta}\left(1+\frac{10}{\delta}\right)|f|_{L^{\infty}}^{2}
\end{gathered}
$$

The induction is complete, and Lemma 4.3 is proved.

For $N$ fixed, we add the relations (4.39) for $n=0, \ldots, N$; this yields

$$
\begin{aligned}
& k \sum_{n=0}^{N}\left\{\left\|y^{n}\right\|^{2}+\left\|z^{n}\right\|^{2}\right\} \\
& \quad \leq \frac{2}{c_{5} \delta}\left(\left|y^{0}\right|^{2}+\left|z^{0}\right|^{2}\right)+\frac{2 k N}{c_{5} \delta}\left(\frac{c_{1}^{2}}{c_{5}}+\frac{10 c_{1}^{2}}{c_{5} \delta}\right)|f|_{L^{\infty}}^{2}=M^{\prime \prime} .
\end{aligned}
$$

We associate with the elements $y_{h}^{n}, z_{h}^{n}$, and $u_{h}^{n}$ the functions $y_{h}, z_{h}$, and $u_{h}$, defined by

$$
\begin{aligned}
& y_{h}: \mathbb{R}_{+} \rightarrow V_{h_{2}}, \quad y_{h}(t)=y_{h}^{n}, \quad t \in[n k,(n+1) k), \\
& z_{h}: \mathbb{R}_{+} \rightarrow W_{h}, \quad z_{h}(t)=z_{h}^{n+1}, \quad t \in[n k,(n+1) k), \\
& u_{h}: \mathbb{R}_{+} \rightarrow V_{h}, \quad u_{h}(t)=y_{h}(t)+z_{h}(t) .
\end{aligned}
$$


In terms of $y_{h}, z_{h}$, and $u_{h}$ we reinterpret the previous results as follows: under the hypotheses (4.8), (4.36), and (4.37),

$$
\begin{aligned}
& \left|y_{h}\right|_{L^{\infty}\left(\mathbb{R}_{+} ; V_{h},|\cdot|_{h}\right)}^{2}+\left|z_{h}\right|_{L^{\infty}\left(\mathbb{R}_{+} ; V_{h},|\cdot|_{h}\right)}^{2} \leq M_{2}, \\
& \left|y_{h}\right|_{L^{2}\left(0, T ; V_{h},\|\cdot\|_{h}\right)}^{2}+\left|z_{h}\right|_{L^{2}\left(0, T ; V_{h},\|\cdot\|_{h}\right)}^{2} \leq M^{\prime \prime} \quad \forall T>0
\end{aligned}
$$

$\left(M_{2}, M^{\prime \prime}\right.$ as in (4.37), (4.42)), and with (4.18),

$$
\begin{aligned}
& u_{h}, y_{h}, \text { and } z_{h} \text { remain bounded in } \\
& L^{\infty}\left(\mathbb{R}_{+} ; V_{h},|\cdot|_{h}\right) \text { and } L^{2}\left(0, T ; V_{h},\|\cdot\|_{h}\right) \\
& \forall T>0, \text { as } k \text { and } h \rightarrow 0 .
\end{aligned}
$$

Scheme III. The first steps of the analysis of Scheme III are the same as those of Scheme II. We replace $\hat{y}_{h}$ by $2 k y_{h}^{n}$ in (3.6) and $\hat{z}_{h}$ by $2 k z_{h}^{n+1}$ in (3.8). The terms corresponding to $z_{h}^{n+1}-z_{h}^{n}$ disappear and thus, in place of (4.33) we have here:

$$
\begin{aligned}
\left|y^{n+1}\right|^{2} & -\left|y^{n}\right|^{2}+2 k a\left(y^{n}+z^{n+1}, y^{n}+z^{n+1}\right) \\
& +2 k d\left(y^{n}+z^{n+1}, y^{n}+z^{n+1}\right) \\
= & 2 k\left(f^{n}, y^{n}+z^{n+1}\right)+\left|y^{n+1}-y^{n}\right|^{2} .
\end{aligned}
$$

The analysis following (4.33) can be repeated without any change, up to (4.39), which now becomes

$$
\begin{aligned}
& \left|y^{n+1}\right|^{2}-\left|y^{n}\right|^{2}+\frac{1}{2} k c_{5} \delta\left(\left\|y^{n}\right\|^{2}+\left\|z^{n+1}\right\|^{2}\right) \\
& \leq k\left(\frac{c_{1}^{2}}{c_{5}}+\frac{10 c_{1}^{2}}{c_{5} \delta}\right)|f|_{L^{\infty}}^{2} .
\end{aligned}
$$

Instead of (4.40) we set

$$
\xi^{n}=\left|y^{n}\right|^{2}+\frac{1}{4} k c_{5} \delta\left(\left\|y^{n}\right\|^{2}+\left\|z^{n+1}\right\|^{2}\right),
$$

and under the hypotheses of Lemma 4.3 we arrive at

$$
\xi^{n+1} \leq\left|y^{0}\right|^{2}+\frac{1}{4} k c_{5} \delta\left\|y^{0}\right\|^{2}+\frac{8 c_{1}^{4}}{c_{5}^{2} \delta}\left(1+\frac{10}{\delta}\right)|f|_{L^{\infty}}^{2} .
$$

Therefore, under the assumptions of Lemma 4.3 we have, in place of (4.38),

$$
\left|y_{h}^{n}\right|_{h}^{2} \leq M_{2} \quad \forall n \geq 0 \text {. }
$$

Then (4.44) remains unchanged, while the first estimate in (4.44) is replaced by

$$
\left|y_{h}\right|_{L^{\infty}\left(\mathbb{R}_{+} ; V_{h},|\cdot|_{h}\right)}^{2} \leq M_{2} \text {. }
$$

In order to recover the estimate of $z_{h}$ in $L^{\infty}\left(\mathbb{R}_{+} ; V_{h},|\cdot|_{h}\right)$, we proceed as follows: we set $\hat{z}_{h}=z_{h}^{n+1}$ in (3.8). This yields

$$
\begin{aligned}
& a\left(z^{n+1}, z^{n+1}\right)+d\left(z^{n+1}, z^{n+1}\right) \\
& \quad=-a\left(y^{n}, z^{n+1}\right)-d\left(y^{n}, z^{n+1}\right)-b\left(y^{n}, y^{n}, z^{n+1}\right)+\left(f^{n}, z^{n+1}\right) .
\end{aligned}
$$


With (1.2), (1.4)-(1.6), and (2.4), we deduce that

$$
\begin{aligned}
c_{5}\left\|z^{n+1}\right\|^{2} \leq & c_{2}\left\|y^{n}\right\|\left\|z^{n+1}\right\|+c_{4}\left\|y^{n}\right\|\left|z^{n+1}\right| \\
& +c_{3}\left|y^{n}\right|^{1 / 2}\left\|y^{n}\right\|^{3 / 2}\left|z^{n+1}\right|^{1 / 2}\left\|z^{n+1}\right\|^{1 / 2}+\left|f^{n}\right|\left|z^{n+1}\right| \\
\leq & \left\{\left(c_{2}+c_{4} S_{2}\right)\left\|y^{n}\right\|+c_{3}\left(S_{2}\right)^{1 / 2}\left|y^{n}\right|^{1 / 2}\left\|y^{n}\right\|^{3 / 2}+S_{2}\left|f^{n}\right|\right\}\left\|z^{n+1}\right\| .
\end{aligned}
$$

Using (2.4) again, and (2.22), we see that

$$
\left|z^{n+1}\right| \leq \frac{1}{c_{5}}\left\{\left(c_{2}+c_{4} S_{2}\right)\left(\frac{S_{2}}{\bar{S}_{1}}\right)\left|y^{n}\right|+\left(\frac{S_{2}}{\bar{S}_{1}}\right)^{3 / 2}\left|y^{n}\right|^{2}+S_{2}^{2}\left|f^{n}\right|\right\}
$$

and thus, for every $n \geq 0$,

$$
\begin{gathered}
\left|z_{h}^{n+1}\right| \leq M_{2}^{\prime}, \\
M_{2}^{\prime}=\frac{1}{c_{5}}\left\{\left(c_{2}+c_{4} S_{2}(h)\right)\left(\frac{S_{2}(h)}{\bar{S}_{1}(h)}\right) M_{2}^{1 / 2}\right. \\
\left.+\left(\frac{S_{2}(h)}{\bar{S}_{1}(h)}\right)^{3 / 2} M_{2}+\left(S_{2}(h)\right)^{2}|f|_{L^{\infty}}\right\} .
\end{gathered}
$$

We define the functions $y_{h}, z_{h}$, and $u_{h}$ exactly as in (4.43) and we state:

$$
\text { under the hypotheses (4.8), (4.36), and (4.37), }
$$

$$
\begin{aligned}
& \left|y_{h}\right|_{L^{\infty}\left(\mathbb{R}_{+} ; V_{h},|\cdot|_{h}\right)}^{2} \leq M_{2}, \quad\left|z_{h}\right|_{L^{\infty}\left(\mathbb{R}_{+} ; V_{h},|\cdot|_{h}\right)} \leq M_{2}^{\prime}, \\
& \left|y_{h}\right|_{L^{2}\left(0, T ; V_{h},\|\cdot\|_{h}\right)}^{2}+\left|z_{h}\right|_{L^{2}\left(0, T ; V_{h},\|\cdot\|_{h}\right)}^{2} \leq M^{\prime \prime} \quad \forall T>0
\end{aligned}
$$

$\left(M_{2}, M^{\prime \prime}, M_{2}^{\prime}\right.$ as in (4.37), (4.42), (4.52)), and with (4.18),

$y_{h}$ remains bounded in $L^{\infty}\left(\mathbb{R}_{+} ; V_{h},|\cdot|_{h}\right)$,

$$
\begin{aligned}
& u_{h}, y_{h}, z_{h} \text { remain bounded in } L^{2}\left(0, T, V_{h},\|\cdot\|_{h}\right) \\
& \forall T \text {, as } k \text { and } h \rightarrow 0 .
\end{aligned}
$$

If, furthermore,

$$
S_{2}(h) / \bar{S}_{1}(h) \text { remains bounded as } h \rightarrow 0
$$

then

$$
u_{h}, z_{h} \text { remain bounded in } L^{\infty}\left(\mathbb{R}_{+} ; V_{h},|\cdot|_{h}\right) \text { as } k \text { and } h \rightarrow 0 \text {. }
$$

Remark 4.4. It follows from (2.4) that, for all the schemes presented here, $z_{h}$ converges to 0 as $k$ and $h$ converge to 0 . We have indeed

$$
\left|z_{h}\right|_{L^{2}\left(0, T ; V_{h},|\cdot|_{h}\right)} \leq S_{2}(h)\left|z_{h}\right|_{L^{2}\left(0, T ; V_{h},\|\cdot\|_{h}\right)} \leq \text { const } \cdot S_{2}(h),
$$

and we recall that $S_{2}(h) \rightarrow 0$ as $h \rightarrow 0$; (4.56) implies that

$$
\left|z_{h}\right|_{L^{2}\left(0, T ; V_{h},|\cdot|_{h}\right)} \rightarrow 0 \text { as } k, h \rightarrow 0, \forall T>0 .
$$


Furthermore, for many examples, the norm $|\cdot|_{h}$ of $V_{h}$ is that of $L^{2}$; then (4.56), (4.57), and the bounds on $z_{h}$ in $L^{\infty}\left(\mathbb{R}_{+} ; V_{h},|\cdot|_{h}\right)$ imply that

$$
\left|z_{h}\right|_{L^{p}\left(0, T ; V_{h},|\cdot|_{h}\right)} \rightarrow 0 \text { as } k, h \rightarrow 0, \forall T>0, \forall p, 1<p<\infty .
$$

As a conclusion to this section we summarize the results that we have proved: for the Schemes I, I', II, III we have obtained some a priori estimates on the $y_{h}^{n}, z_{h}^{n}$ which demand some restrictive conditions on $k$ and $h$, namely the classical stability conditions. The a priori estimates are completed and reinterpreted for the different schemes in the statements (4.20)-(4.23), (4.31) and (4.32), (4.44) and (4.45), and finally for Scheme III in (4.53)-(4.55) (see also (4.56), (4.57) in Remark 4.4).

\section{APPLICATIONS}

The object of this section is to make explicit the hypotheses and results of the previous sections on some examples. For the space discretizations we will consider those of $\S 2$ and also some finite elements and finite differences in space dimension 2; two evolution equations of the Navier-Stokes type will be considered.

We start by making explicit the constants $S_{1}(h), \bar{S}_{1}(h)$, and $S_{2}(h)$ for various spaces. In particular, we show that the ratio

$$
S_{2}(h) / \bar{S}_{1}(h)
$$

that appears in the stability conditions for certain schemes remains bounded.

(i) Spectral discretization. The framework is that of $\S 2$. We have seen in (2.6) that

$$
S_{2}(h)=\left(\lambda_{m_{2}+1}\right)^{-1 / 2} .
$$

For estimating $S_{1}$, we note that, for every $u_{h}$ in $V_{h}, u_{h}=\sum_{j=1}^{m_{1}} \xi_{j} w_{j}$, and we have

$$
\left\|u_{h}\right\|_{h}^{2}=\left\|\sum_{j=1}^{m_{1}} \xi_{j} w_{j}\right\|^{2}=\sum_{j=1}^{m_{1}} \xi_{j}^{2} \lambda_{j} \leq \lambda_{m_{1}} \sum_{j=1}^{m_{1}} \xi_{j}^{2}=\lambda_{m_{1}}\left|u_{h}\right|^{2}=\lambda_{m_{1}}\left|u_{h}\right|_{h}^{2} .
$$

Thus,

$$
S_{1}(h)=\left(\lambda_{m_{1}}\right)^{-1 / 2} \text {. }
$$

Similarly, for $\bar{S}_{1}$ we just replace $m_{1}$ by $m_{2}$ in (5.3):

$$
\bar{S}_{1}(h)=\left(\lambda_{m_{2}}\right)^{-1 / 2} \text {. }
$$

We have

$$
S_{2} / \bar{S}_{1}=\left(\lambda_{m_{2}} / \lambda_{m_{2}+1}\right)^{1 / 2} \leq 1
$$

and this ratio is obviously bounded as $m_{i} \rightarrow \infty$.

Some indication of the advantage of using decompositions of the space $V_{h}$ of type (2.1) can be obtained by comparing the stability conditions appearing 
above with those appearing when no decomposition is used, i.e., $W_{h}=\{0\}$, $S_{1}=\bar{S}_{1}$.

For example, for the case of the Dirichlet problem in space dimension $D$,

$$
\lambda_{m} \sim \mathrm{cm}^{2 / D} \text { as } m \rightarrow \infty .
$$

If we consider a decomposition with $m_{1}=4 m_{2}$, then

$$
\bar{S}_{1} \sim c m_{2}^{-1 / D}, \quad S_{1} \sim c\left(4 m_{2}\right)^{-1 / D}, \quad\left(\bar{S}_{1} / S_{1}\right)^{2} \sim 4^{2 / D},
$$

so that Scheme $I^{\prime}$, for example (see (4.26)), allows a time step $4^{2 / D}$ times larger. Note that this is only an indication about stability, since the stability conditions are only sufficient ones. However, such improvements can be rigorously proved in the linear case, where the stability conditions are necessary and sufficient [24]; they are also broadly supported by numerical experiments $[5,13,14]$.

(ii) Finite elements $(1 D), \mathscr{P}_{1}$-finite elements in space dimension one have been discussed in $\S 2$. With, for instance, $h_{2}=2 h_{1}=2 h$, we have seen that

$$
S_{1}(h)=h / 2 \sqrt{3}, \quad S_{2}(h)=h / \sqrt{3} .
$$

Of course, replacing $h$ by $h_{2}$,

$$
\bar{S}_{1}(h)=h / \sqrt{3} \text { and } S_{2}(h) / \bar{S}_{1}(h)=1,
$$

so that this ratio is obviously bounded. In the present case,

$$
\left(\bar{S}_{1}(h) / S_{1}(h)\right)^{2}=4,
$$

and for Schemes I' , II, or III, the stability conditions (4.26), (4.36), (4.37) allow a time step four times larger when this decomposition is used.

(iii) Finite differences $(1 D)$. We have seen in $\S 2$ that

$$
S_{1}(h)=h / 2, \quad S_{2}(h)=h .
$$

Then, by Remark 2.1,

$$
\bar{S}_{1}(h)=S_{1}(2 h) / \sqrt{2}=h / \sqrt{2} \text { and } S_{2}(h) / \bar{S}_{1}(h)=\sqrt{2},
$$

so that this ratio is indeed bounded (constant) as $h \rightarrow 0$.

For $h_{1}=h=2 h_{2}$,

$$
\left(\bar{S}_{1}(h) / S_{1}(h)\right)^{2}=2,
$$

and for schemes I', II, or III the stability conditions (4.26), (4.36), (4.37) allow a time step two times larger when this decomposition is used.

(iv) Finite elements $(2 D)$. In space dimension 2, decompositions of the space $V_{h}$ based on $\mathscr{P}_{1}$-elements and other finite elements have been studied in [19]. The description of the spaces $V_{h}, V_{h_{2}}, W_{h}$ will not be reproduced here. We only borrow from [19] (see (3.10)) the fact that $S_{2}(h)=\rho_{h}$, where $\rho_{h}$ is the supremum of the diameters of the triangles in the corresponding triangulation $\mathscr{T}_{h}$. One can show also (see $\left[21\right.$, p. 376]) that $S_{1}(h)$ is of the form $S_{1}(h)=c \rho_{h}^{\prime}$, 
where $\rho_{h}^{\prime}$ is the smallest of the diameters of the inscribed circles in the triangles $T$ of the triangulation $\mathscr{T}_{h}$.

If the triangulation $\mathscr{T}_{h}$ is associated with the triangulation $\mathscr{T}_{h}$ by dividing each triangle $T$ of $\mathscr{T}_{h_{2}}$ into four equal triangles (congruent to $T$ ), then

$$
\bar{S}_{1}(h)=S_{1}(2 h)=c \rho_{2 h}^{\prime}=2 c \rho_{h}^{\prime}=2 S_{1}(h) \text {. }
$$

Thus,

$$
S_{2}(h) / \bar{S}_{1}(h)=\rho_{h} /\left(2 c \rho_{h}^{\prime}\right),
$$

and this quantity remains usually bounded as $h \rightarrow 0$, although this is not an automatic assumption. Also, we note that $\left(\bar{S}_{1}(h) / S_{1}(h)\right)^{2}=4$.

(v) Finite differences $(2 D)$. Decomposition of the space $V_{h}$ for finite differences in space dimension two have been presented in [24]. It was shown (see Lemma 2.3 in [24]) that $S_{2}(h)=h$, where $h>0$ is the space discretization mesh in both directions. Also (see (5.17) in [24]), $S_{1}(h)=h / 2 \sqrt{2}$, and by Lemma 2.2 in [24]: $\bar{S}_{1}(h)=h / 2$. Hence, $S_{2}(h)=2 \bar{S}_{1}(h)$, and this ratio is constant, thus bounded. We have

$$
\left(\bar{S}_{1}(h) / S_{1}(h)\right)^{2}=2 .
$$

We conclude this section by describing two equations which lead to an equation of the form (1.7) by space discretization. As mentioned before, the NavierStokes equations, and the thermohydraulic and magnetohydrodynamic equations in space dimension two lead to equations of the form (1.7), but these specific and very important cases will be treated in a separate work.

Example 1. Burger's equation. Let $\Omega=(0, L), L>0$; we set $V=H_{0}^{1}(0, L)$, $H=L^{2}(0, L)$. For $\nu>0$ and $f$ given, the equation is written as

$$
\begin{gathered}
\frac{\partial u}{\partial t}-\nu \frac{\partial^{2} u}{\partial x^{2}}+u \frac{\partial u}{\partial x}=f \quad \text { in } \Omega \times \mathbb{R}_{+}, \\
u(0, t)=u(L, t)=0, \quad u(x, t)=u_{0}(x) .
\end{gathered}
$$

The variational form of this evolution equation consists in finding a function $u: \mathbb{R}_{+} \rightarrow H_{0}^{1}(\Omega)=V$ such that

$$
\frac{d}{d t}(u, v)+\nu((u, v))+b(u, u, v)=(f, v) \quad \forall v \in V .
$$

Here,

$$
(\varphi, \psi)=\int_{0}^{L} \varphi \psi d x, \quad((\varphi, \psi))=\int_{0}^{L} \frac{d \varphi}{d x} \frac{d \psi}{d x} d x \quad \forall \varphi, \psi,
$$

and $b$ is the antisymmetrized form of the nonlinear term [20]

$$
b(\varphi, \psi, \theta)=\frac{1}{3} \int_{0}^{L} \varphi\left(\frac{d \psi}{d x} \theta-\psi \frac{d \theta}{d x}\right) d x .
$$


By using a discretization of (5.5) by any of the methods (i), (ii), (iii), we obtain an equation of the form (1.7) with $d=0$. The hypotheses (1.2)-(1.6) are satisfied, $c_{2}=c_{5}=\nu, c_{4}=0$, and $c_{3}$ is an appropriate constant.

Example 2. An equation of Navier-Stokes type. Let $\Omega \subset \mathbb{R}^{2}$ be an open bounded set. We consider a modified form of the Navier-Stokes equations where the pressure and the divergence-free conditions have been removed, while the nonlinear term is modified as in [20]. Namely, $u=u(x, t)$ satisfies

$$
\begin{gathered}
\frac{\partial u}{\partial t}-\nu \Delta u+(u \cdot \nabla) u+\frac{1}{2}(\operatorname{div} u) u=f, \quad x \in \Omega, t>0, \\
u=0 \quad \text { on } \partial \Omega, \quad u(x, 0)=u_{0}(x) .
\end{gathered}
$$

This problem is equivalent to finding a function $u: \mathbb{R}_{+} \rightarrow V=H_{0}^{1}(\Omega)^{2}$ satisfying an equation similar to (5.5), now with

$$
\begin{gathered}
(\varphi, \psi)=\int_{\Omega} \varphi \cdot \psi d x, \quad((\varphi, \psi))=\sum_{i, j=1}^{2} \int_{\Omega} \frac{\partial \varphi_{i}}{\partial x_{j}} \frac{\partial \psi_{i}}{\partial x_{j}} d x, \\
b(\varphi, \psi, \theta)=\frac{1}{2} \sum_{i, j=1}^{2} \int_{\Omega} \varphi_{i}\left(\frac{\partial \psi_{j}}{\partial x_{i}} \theta_{j}-\psi_{j} \frac{\partial \theta_{j}}{\partial x_{i}}\right) d x
\end{gathered}
$$

By using a discretization of (5.5) by one of the methods (i), (iv), (v) above, we obtain an equation of the form (1.7) with $d=0$. The hypotheses (1.2)-(1.6) are satisfied, and again $c_{2}=c_{5}=\nu, c_{4}=0$, and $c_{3}$ is an appropriate constant.

\section{BIBLIOGRAPHY}

1. O. Axelsson and I. Gustafsson, Preconditioning and two-level multigrid methods of arbitrary degree of approximation, Math. Comp. 40 (1983), 219-242.

2. C. Canuto, M. Y. Hussaini, A. Quarteroni, and T. A. Zang, Spectral methods in fluid dynamics, Springer-Verlag, New York, 1988.

3. J. Céa, Approximation variationnelle des problèmes aux limites, Ann. Inst. Fourier (Grenoble) 14 (1964), 345-444.

4. P. G. Ciarlet, The finite element method for elliptic problems, North-Holland, Amsterdam, 1978.

5. T. Dubois, F. Jauberteau, and R. Temam, The nonlinear Galerkin method for the two and three dimensional Navier-Stokes equations (to appear).

6. C. Foiaş, M. S. Jolly, I. G. Kevrekidis, G. R. Sell, and E. S. Titi, On the computation of inertial manifolds, Phys. Lett. A 131 (1988), 433-436.

7. C. Foiaş, O. Manley, and R. Temam, Sur l'interaction des petits et grands tourbillons dans les écoulements turbulents, C.R. Acad. Sci. Paris Sér. I Math. 305 (1987), 497-500; On the interaction of small and large eddies in two-dimensional turbulent flows, Math. Mod. Numer. Anal. 22 (1988), 93-114.

8. C. Foias, B. Nicolaenko, G. Sell, and R. Temam, Inertial manifolds for the KuramotoSivashinsky equation and an estimate of their lowest dimension, J. Math. Pures Appl. 67 (1988), 197-226.

9. C. Foiaş, G. R. Sell, and R. Temam, Inertial manifolds for nonlinear evolutionary equations, J. Differential Equations 73 (1988), 309-353. 
10. C. Foiaş, G. Sell, and E. Titi, Exponential tracking and approximation of inertial manifolds for dissipative nonlinear equations, J. Dynamical Differential Equations 1 (1989), 199-224.

11. D. Gottlieb and S. A. Orszag, Numerical analysis of spectral methods: Theory and applications, Regional Conf. Ser. in Appl. Math., SIAM, Philadelphia, PA, 1977.

12. J. Hale, Asymptotic behavior of dissipative systems, Math. Surveys and Monographs, vol. 25, Amer. Math. Soc., Providence, RI, 1988.

13. F. Jauberteau, C. Rosier, and R. Temam, The nonlinear Galerkin method in computational fluid dynamics, Appl. Numer. Math. 6 (1989/90), 361-370.

14. __ A nonlinear Galerkin method for the Navier-Stokes equations, Comput. Methods Appl. Mech. Engrg. 80 (1990), 245-260.

15. M. S. Jolly, I. G. Kevrekidis, and E. S. Titi, Approximate inertial manifolds for the KuramotoSivashinsky equation: analysis and computation, preprint, 1990.

16. __, Preserving dissipation in approximate inertial forms, preprint, 1990.

17. J. Mallet-Paret and G. R. Sell, Inertial manifolds for reaction diffusion equations in higher space dimensions, J. Amer. Math. Soc. 1 (1988), 805-866.

18. M. Marion and R. Temam, Nonlinear Galerkin methods, SIAM J. Numer. Anal. 26 (1989), 1139-1157.

19. __ Nonlinear Galerkin methods: The finite elements case, Numer. Math. 57 (1990), 205226.

20. R. Temam, Sur l'approximation des équations de Navier-Stokes, C.R. Acad. Sci. Paris Sér. A 262 (1966), 219-221.

21. _ Navier-Stokes equations, North-Holland, Amsterdam, 1984.

22. __ Induced trajectories and approximate inertial manifolds, Math. Mod. Numer. Anal. 23 (1989), 541-561.

23. __ Attractors for the Navier-Stokes equations, localization and approximation, J. Fac. Sci. Univ. Tokyo Sect. IA Math. 36 (1989), 629-647.

24. __ Inertial manifolds and multigrid methods, SIAM J. Math. Anal. 21 (1990), 154-178.

25. __ Infinite dimensional dynamical systems in mechanics and physics, Appl. Math. Sci., vol. 68, Springer-Verlag, New York, 1988.

26. E. S. Titi, Une variété approximante de l'attracteur universel des équations de Navier-Stokes non linéaires de dimension finie, C.R. Acad. Sci. Paris Sér. I Math. 307 (1988), 383-385.

Laboratoire d'Analyse Numérique, Universite Paris-Sud, Bât. 425, 91405 Orsay, FRANCE 